\title{
Master integrals for the NNLO virtual corrections to $\mu e$ scattering in QED: the non-planar graphs
}

\author{
Stefano Di Vita, ${ }^{a}$ Stefano Laporta, ${ }^{b, c}$ Pierpaolo Mastrolia, ${ }^{b, c}$ Amedeo Primo ${ }^{d}$ \\ and Ulrich Schubert ${ }^{e}$ \\ ${ }^{a}$ INFN, Sezione di Milano, \\ Via Celoria 16, 20133 Milano, Italy \\ ${ }^{b}$ Dipartimento di Fisica ed Astronomia, Università di Padova, \\ Via Marzolo 8, 35131 Padova, Italy \\ ${ }^{c}$ INFN, Sezione di Padova, \\ Via Marzolo 8, 35131 Padova, Italy \\ ${ }^{d}$ Department of Physics, University of Zürich, \\ CH-8057 Zürich, Switzerland \\ ${ }^{e}$ High Energy Physics Division, Argonne National Laboratory, \\ Argonne, IL 60439, U.S.A. \\ E-mail: stefano.divita@mi.infn.it, stefano.laporta@pd.infn.it, \\ pierpaolo.mastrolia@pd.infn.it, aprimo@physik.uzh.ch, \\ schubertmielnik@anl.gov
}

ABSTRACT: We evaluate the master integrals for the two-loop non-planar box-diagrams contributing to the elastic scattering of muons and electrons at next-to-next-to-leading order in QED. We adopt the method of differential equations and the Magnus exponential to determine a canonical set of integrals, finally expressed as a Taylor series around four spacetime dimensions, with coefficients written as combination of generalised polylogarithms. The electron is treated as massless, while we retain full dependence on the muon mass. The considered integrals are also relevant for crossing-related processes, such as di-muon production at $e^{+} e^{-}$colliders, as well as for the QCD corrections to top-pair production at hadron colliders. In particular our results, together with the planar master integrals recently computed, represent the complete set of functions needed for the evaluation of the photonic two-loop virtual next-to-next-to-leading order QED corrections to $\mu e \rightarrow \mu e$ and $e^{+} e^{-} \rightarrow \mu^{+} \mu^{-}$.

KEYWORDS: NLO Computations

ArXiv EPrint: 1806.08241 


\section{Contents}

1 Introduction 1

2 The non-planar four-point topology 4

3 System of differential equations 5

$\begin{array}{ll}3.1 \text { Boundary conditions } & 10\end{array}$

4 Numerical evaluation of the non-planar four point integrals 11

4.1 The non-planar box in $d=6$ dimensions $\quad 12$

$\begin{array}{lll}4.1 .1 & \text { Analytic integrations } & 13\end{array}$

$\begin{array}{ll}\text { 4.1.2 Numerical integrations } & 14\end{array}$

5 Conclusions $\quad 16$

A Evaluation of the auxiliary vertex integrals for eq. (3.18) 17

$\begin{array}{ll}\text { B } d \log \text { forms } & 19\end{array}$

\section{Introduction}

In a previous work [1], we began the investigation of the next-to-next-to-leading-order (NNLO) virtual corrections to the elastic scattering of muons and electrons in Quantum Electrodynamics (QED), by classifying and evaluating the planar two-loop integrals arising from Feynman diagrams at this order in perturbation theory [2].

The NNLO QED corrections to the process $\mu e \rightarrow \mu e$ are crucial to interpret the highprecision data of future experiments like MUonE, recently proposed at CERN, aiming at measuring the differential cross section of the elastic scattering of high-energy muons on atomic electrons as a function of the spacelike (negative) squared momentum transfer [3, 4]. This measurement will provide the running of the effective electromagnetic coupling in the spacelike region and, as a result, a new and independent determination of the leading hadronic contribution to the muon $g-2[3,4]$. In order for this new determination to be competitive with the present dispersive one, which is obtained via timelike data (see [5] for a review), the $\mu e$ differential cross section must be measured with statistical and systematic uncertainties of the order of 10ppm. This high experimental precision demands an analogous accuracy in the theoretical prediction.

Moreover, the NNLO QED corrections for the crossing-related scattering process $e^{+} e^{-}$ $\rightarrow \mu^{+} \mu^{-}$are important for some of the high-precision studies planned at upcoming lowenergy $e^{+} e^{-}$experiments, like Belle-II and VEPP-2000. Two interesting applications would be the following. The forward-backward asymmetry in muon pair production could be 
exploited to constrain non-standard ee $\mu \mu$ interactions [6], and the current estimates suggest that the knowledge of the NNLO QED differential cross section is needed, as QED itself produces an asymmetry starting at NLO. The knowledge of the QED radiative corrections to the $e^{+} e^{-} \rightarrow \mu^{+} \mu^{-}$cross section will also be needed for precise measurements of the ratio $R(s)=\sigma\left(e^{+} e^{-} \rightarrow\right.$ hadrons $) / \sigma\left(e^{+} e^{-} \rightarrow \mu^{+} \mu^{-}\right)[7,8]$.

In this work, we complete the task of determining all functions required by the NNLO QED virtual photonic corrections to $\mu$ e scattering, by evaluating the two-loop integrals coming from non-planar four-point Feynman diagrams. Given the hierarchy between the electron mass $m_{e}$ and the muon mass $m, m_{e} / m \sim 5 \cdot 10^{-3}$, as in our former study we consider the approximation $m_{e}=0 .{ }^{1}$ For the non-planar topology, integration-by-parts identities (IBPs) [11-13] yield the identification of a set of 44 master integrals (MIs), which we compute analytically by means of the differential equations method [14-16]. The system-solving strategy $[17,18]$ is based on a consolidated procedure, which has been proven to be very effective in the context of multi-loop integrals involving several scales [1, 18-21]. Firstly, we identify a set of MIs that obey a system of first-order differential equations (DEQs) in the kinematic variables $s / m^{2}$ and $t / m^{2}$ which is linear in the space-time dimension $d$. Subsequently we employ the Magnus exponential matrix [18] to derive an equivalent system of equations in canonical form [17], where the dependence of the associated matrices on $(d-4)$ is factorised from the kinematics. The matrix associated with the canonical system is a logarithmic differential form which, in appropriate variables, has a polynomial alphabet. The canonical MIs can be therefore cast as a Taylor series around $d=4$, with coefficients written as combinations of generalised polylogarithms (GPLs) [22-25].

For certain classes of MIs, like the ones of $\mu e \rightarrow \mu e$ and crossing-related processes, the choice of the boundary conditions may also constitute a challenging problem. Here, we exploit either the regularity conditions at pseudo-thresholds or the expression of integrals which are obtained by solving simpler, auxiliary systems of DEQs. Therefore, we limit the use of direct integration only to a small subset of simpler integrals used as input functions.

The package Reduze [26] has been used throughout the calculations, for the IBPs decomposition and for generating the DEQs obeyed by the MIs. The analytic expressions of the MIs have been numerically evaluated with the help of GiNaC [27] and were successfully tested against the numerical values provided either by the computer code SecDec [28] or, for the most complicated two-loop non-planar topologies (with 6- and 7-denominators), by an in-house algorithm. For such topologies, we identified an alternative set of quasi-finite integrals [29], more suitable for numerical integration, also with the help of Reduze.

As far as the QED corrections involving two leptons (one massless, one massive) are concerned, the non-planar four-point functions hereby presented, together with the planar

\footnotetext{
${ }^{1}$ The simplifying assumption $m_{e}=0$ has already been used in the case of the two-loop photonic corrections to Bhabha scattering [9], with which $\mu e$ scattering shares several features. In particular, one expects to find $\log$ arithmically enhanced terms, proportional to $\log \left(s / m_{e}^{2}\right)$ and $\log ^{2}\left(s / m_{e}^{2}\right)$, in the cross-sections for such processes. Such terms would correspond to collinear singularities in the $m_{e} \rightarrow 0$ limit. For Bhabha scattering it was possible to precisely recover the coefficients of those terms, starting from the calculation in which the collinear divergenes are dimensionally regulated, and exploiting the universal infrared structure of gauge theories (see [10] and references therein).
} 
ones presented earlier [1], and the three-point functions available in the literature [30-32], make the analytic evaluation of the virtual two-loop amplitudes for the $\mu e$ scattering, as well as for the crossing-related processes, within reach. In order to build the corresponding two-loop virtual amplitude, one would still need to compute the corresponding Feynman diagrams by reducing them to our set of MIs and carrying out the ultraviolet renormalization. Finally, the analytic continuation to the $\mu e$ scattering region $\left(s \geq m^{2},-\left(m^{2}-s\right)^{2} \leq s t \leq 0\right.$, and $\left.s\left(2 m^{2}-s\right) \leq s u \leq m^{4}\right)$ has to be performed. We leave all these steps to a future publication.

For completeness we mention that, as in the case of Bhabha scattering (see e.g. ref. [10] for a review), further gauge-invariant sets of NNLO corrections to $\mu e$ scattering will have to be computed. Those sets consist of vertex and box diagrams with vacuum polarization insertions of two kinds: $i$ ) vacuum polarization due to a heavy-fermion loop (the tau lepton or the top, bottom and charm quarks), which can be addressed perturbatively at energies much lower than their masses, the only difference being the presence of a further scale in the loop integrals; $i$ ) vacuum polarization due a light-quark loop, where the effect of the hadronic interactions cannot be neglected and a non-perturbative approach, such as the use of dispersion relations, is required.

In addition, we remark that the MIs of the QED corrections to $e^{+} e^{-} \rightarrow \mu^{+} \mu^{-}$are a subset of those needed for the QCD corrections to the $t \bar{t}$-pair production at hadron colliders. The complete two-loop QCD corrections to $p p \rightarrow t \bar{t}$ are currently known only numerically [33-37]. The analytic evaluation of the MIs appearing in the leading-colour corrections to $p p \rightarrow t \bar{t}$ were considered in refs. [38-42], which also include the simplest nonplanar topology, namely the one in which the crossed-loop is fully massless. In our former work [1], we extended the set of available functions for considering also sub-leading colour contributions. Very recently, the analytic calculation of the MIs for the planar double-box integral with a closed top loop appeared in [43]. The analytic result for a non-planar threepoint function, which constitutes a sub-diagram of the non-planar double box with closed heavy quark loop, was presented in [44]. The non-planar graphs hereby considered would also contribute to subleading-colour terms to $t \bar{t}$-pair production, and their analytic evaluation was never addressed before. The numerical evaluation of (one of the) non-planar integrals computed analytically in this work has been recently considered in [45], in the context of a novel, promising method that aims at the numerical solution of differential equations.

The paper is organised as follows. In section 2, we set our notation and conventions for the four-point topology relevant for $\mu e$ scattering. In section 3, we describe the general features of the systems of DEQs satisfied by the MIs, cast in $d \log$-form, and we present the results for the non-planar two-loop MIs. In section 4, we describe the numerical evaluation of the non-planar four-point integrals. The information provided in the text is complemented by two appendices: in appendix A, we discuss the computation of the auxiliary integrals which have been used to extract some of the boundary constants and, in appendix B, we give the matrices associated with the $d \log$-form.

The analytic expressions of the considered MIs are given in the ancillary files accompanying the arXiv version of this publication. 


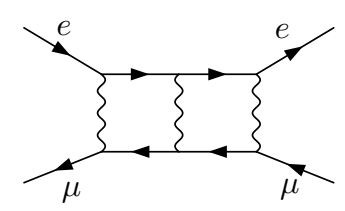

$T_{1}$

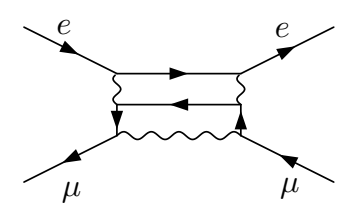

$T_{4}$

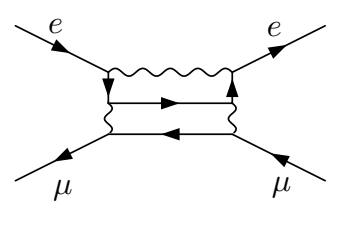

$T_{2}$

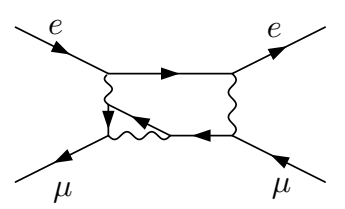

$T_{5}$

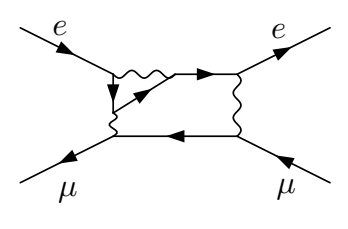

$T_{3}$

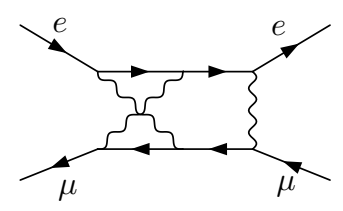

$T_{6}$

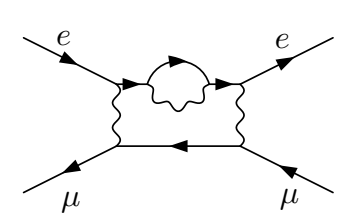

$T_{7}$

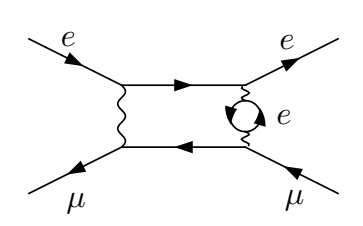

$T_{8}$

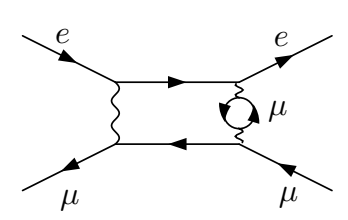

$T_{9}$

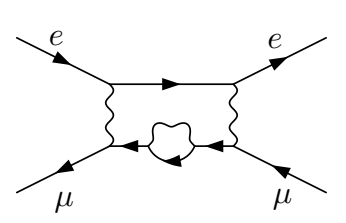

$T_{10}$

Figure 1. Two-loop four-point topologies for $\mu e$ scattering.

\section{The non-planar four-point topology}

In this paper, we consider the $\mu e$ scattering process

$$
\mu^{+}\left(p_{1}\right)+e^{-}\left(p_{2}\right) \rightarrow e^{-}\left(p_{3}\right)+\mu^{+}\left(p_{4}\right)
$$

in the approximation of vanishing electron mass, $m_{e}=0$, i.e. with kinematics specified by

$$
\begin{aligned}
p_{1}^{2} & =p_{4}^{2}=m^{2}, \quad p_{2}^{2}=p_{3}^{2}=0, \\
s & =\left(p_{1}+p_{2}\right)^{2}, \quad t=\left(p_{2}-p_{3}\right)^{2}, \quad u=\left(p_{1}-p_{3}\right)^{2}=2 m^{2}-t-s,
\end{aligned}
$$

where $m$ is the muon mass. Representative Feynman diagrams of the 10 relevant twoloop four-point topologies $T_{i}$ that contribute to the process are depicted in figure 1 . The computation of the MIs belonging to the topologies $T_{1,2,3,4,5,7,8,9,10}$ has been discussed in [1]. In this paper, we complete the evaluation of all MIs required for the two-loop virtual amplitude due to photonic corrections, by determining the analytic expression of the MIs that belong to the non-planar topology $T_{6}$. 
The calculation involves the evaluation, in $d$ dimensions, of Feynman integrals of the type

$$
I^{[d]}\left(n_{1}, \ldots, n_{9}\right) \equiv \int \widetilde{\mathrm{d}^{d} k_{1}} \widetilde{\mathrm{d}^{d} k_{2}} \frac{1}{D_{1}^{n_{1}} \ldots D_{9}^{n_{9}}},
$$

where $D_{i}$ are inverse scalar propagators. The analytic calculation described in section 3 is performed expanding around $d=4$, while the numerical evaluation presented in section 4 is carried over around $d=6$. We set $\epsilon \equiv\left(d_{*}-d\right) / 2$, where $d_{*}=4$ and $d_{*}=6$ according to the case considered, and define our integration measure

$$
\widetilde{\mathrm{d}^{d} k}=\frac{\mathrm{d}^{d} k}{i \pi^{d / 2} \Gamma_{\epsilon}}\left(\frac{m^{2}}{\mu^{2}}\right)^{\epsilon}
$$

where $\mu$ is the 't Hooft scale of dimensional regularisation and $\Gamma_{\epsilon} \equiv \Gamma(1+\epsilon)$. Notice that our integration measure, when evaluated at $d=4-2 \epsilon$, agrees with eq. (3.2) of [1].

For the non-planar four-point topology $T_{6}$, we choose the following set of inverse propagators:

$$
\begin{array}{llll}
D_{1}=\left(k_{1}\right)^{2}-m^{2}, & D_{2}=\left(k_{2}\right)^{2}-m^{2}, & D_{3}=\left(k_{1}+p_{1}\right)^{2}, & D_{4}=\left(k_{2}+p_{1}\right)^{2}, \\
D_{5}=\left(k_{1}+p_{1}+p_{2}\right)^{2}, & D_{6}=\left(k_{2}+p_{1}+p_{2}\right)^{2}, & D_{7}=\left(k_{1}-k_{2}\right)^{2}, & \\
D_{8}=\left(k_{2}+p_{1}+p_{2}-p_{3}\right)^{2}, & D_{9}=\left(k_{1}-k_{2}+p_{3}\right)^{2}, &
\end{array}
$$

where $k_{1}$ and $k_{2}$ denote the loop momenta. In particular, with the definition (2.4), the tadpole integral $\epsilon^{2} I^{[4-2 \epsilon]}(2,2,0, \ldots, 0)$ is normalised to 1 . The Feynman prescription is understood, and it can be recovered by the addition of an arbitrarily small positive imaginary part, $D_{i} \rightarrow D_{i}+i \omega$.

\section{System of differential equations}

By means of IBPs, the two-loop integrals that belong to $T_{6}$ can be reduced to a basis of 44 distinct MIs. In order to determine the analytic expression of the latter, we derive their DEQs in the kinematic variables $s$ and $t$. The evaluation of the MIs can be further facilitated by parametrising the Mandelstam invariants in term of two independent dimensionless variables, $w$ and $z$, which are defined by

$$
\frac{u-m^{2}}{s-m^{2}}=-\frac{z^{2}}{w}, \quad \frac{t}{m^{2}}=-\frac{(1-w)^{2}}{w},
$$

where the constraint $s+t+u=2 m^{2}$ is understood. Such change of variables rationalises the canonical DEQs. ${ }^{2}$

${ }^{2}$ At an earlier stage of the project, we found that the variables $x$ and $y$, defined through,

$$
\frac{s-m^{2}}{m^{2}}=-x^{2}\left(1-\frac{\left(1-y^{2}\right)^{2}}{4 x^{2} y^{2}}\right)^{2}, \quad \frac{t}{m^{2}}=-\frac{\left(1-y^{2}\right)^{2}}{y^{2}},
$$

remove all irrational terms appearing in the system of DEQs, individually. However, as pointed out by Lorenzo Tancredi - whom we acknowledge for the suggestion -, it is sufficient to rationalise just those combinations of irrational terms that appear in the DEQs, by means of $w$ and $z$ defined through in eq. (3.1), to yield a polynomial alphabet. 
A canonical basis of MIs in $d=4-2 \epsilon$ can be identified by making use of the algorithm described in $[18,19]$. Namely, we start by choosing an initial set of MIs $F_{i}$ that fulfill DEQs with linear dependence on the dimensional regularisation parameter $\epsilon$,

$$
\begin{aligned}
& \mathrm{F}_{1}=\epsilon^{2} \mathcal{T}_{1}, \quad \mathrm{~F}_{2}=\epsilon^{2} \mathcal{T}_{2}, \quad \mathrm{~F}_{3}=\epsilon^{2} \mathcal{T}_{3}, \\
& \mathrm{~F}_{4}=\epsilon^{2} \mathcal{T}_{4}, \quad \mathrm{~F}_{5}=\epsilon^{2} \mathcal{T}_{5}, \quad \mathrm{~F}_{6}=\epsilon^{2} \mathcal{T}_{6}, \\
& \mathrm{~F}_{7}=\epsilon^{2} \mathcal{T}_{7}, \quad \mathrm{~F}_{8}=\epsilon^{2} \mathcal{T}_{8}, \quad \mathrm{~F}_{9}=\epsilon^{3} \mathcal{T}_{9}, \\
& \mathrm{~F}_{10}=\epsilon^{3} \mathcal{T}_{10}, \quad \mathrm{~F}_{11}=\epsilon^{3} \mathcal{T}_{11}, \quad \mathrm{~F}_{12}=\epsilon^{2} \mathcal{T}_{12}, \\
& \mathrm{~F}_{13}=\epsilon^{3} \mathcal{T}_{13}, \quad \mathrm{~F}_{14}=\epsilon^{2} \mathcal{T}_{14}, \quad \mathrm{~F}_{15}=\epsilon^{3} \mathcal{T}_{15}, \\
& \mathrm{~F}_{16}=\epsilon^{2} \mathcal{T}_{16}, \quad \mathrm{~F}_{17}=\epsilon^{2} \mathcal{T}_{17}, \quad \mathrm{~F}_{18}=\epsilon^{4} \mathcal{T}_{18}, \\
& \mathrm{~F}_{19}=\epsilon^{3} \mathcal{T}_{19}, \quad \mathrm{~F}_{20}=\epsilon^{4} \mathcal{T}_{20}, \quad \mathrm{~F}_{21}=\epsilon^{2}(1+2 \epsilon) \mathcal{T}_{21}, \\
& \mathrm{~F}_{22}=\epsilon^{3} \mathcal{T}_{22}, \quad \mathrm{~F}_{23}=\epsilon^{4} \mathcal{T}_{23}, \quad \mathrm{~F}_{24}=\epsilon^{3} \mathcal{T}_{24}, \\
& \mathrm{~F}_{25}=\epsilon^{4} \mathcal{T}_{25}, \quad \mathrm{~F}_{26}=\epsilon^{3} \mathcal{T}_{26}, \quad \mathrm{~F}_{27}=\epsilon^{3} \mathcal{T}_{27}, \\
& \mathrm{~F}_{28}=\epsilon^{2} \mathcal{T}_{28}, \quad \mathrm{~F}_{29}=\epsilon^{4} \mathcal{T}_{29}, \quad \mathrm{~F}_{30}=\epsilon^{3} \mathcal{T}_{30}, \\
& \mathrm{~F}_{31}=\epsilon^{4} \mathcal{T}_{31}, \quad \mathrm{~F}_{32}=\epsilon^{3} \mathcal{T}_{32}, \quad \mathrm{~F}_{33}=\epsilon^{3} \mathcal{T}_{33}, \\
& \mathrm{~F}_{34}=\epsilon^{3} \mathcal{T}_{34}, \quad \mathrm{~F}_{35}=\epsilon^{4} \mathcal{T}_{35}, \quad \mathrm{~F}_{36}=\epsilon^{3}(1-2 \epsilon) \mathcal{T}_{36}, \\
& \mathrm{~F}_{37}=\epsilon^{4} \mathcal{T}_{37}, \quad \mathrm{~F}_{38}=\epsilon^{4} \mathcal{T}_{38}, \quad \mathrm{~F}_{39}=\epsilon^{4} \mathcal{T}_{39}, \\
& \mathrm{~F}_{40}=\epsilon^{4} \mathcal{T}_{40}, \quad \mathrm{~F}_{41}=\epsilon^{4} \mathcal{T}_{41}, \quad \mathrm{~F}_{42}=\epsilon^{4} \mathcal{T}_{42}, \\
& \mathrm{~F}_{43}=\epsilon^{4} \mathcal{T}_{43}, \quad \mathrm{~F}_{44}=\epsilon^{4} \mathcal{T}_{44},
\end{aligned}
$$

where the $\mathcal{T}_{i}$ are the integrals depicted in figure 2 .

Subsequently, we use the Magnus exponential in order rotate the integrals of eq. (3.2) into a new basis of MIs $\mathrm{I}_{i}$ that satisfy canonical DEQs in both variables $w$ and $z$ (or, equivalently, in $s$ and $t$ ),

$$
\begin{aligned}
& \mathrm{I}_{1}=\mathrm{F}_{1}, \\
& \mathrm{I}_{2}=-s \mathrm{~F}_{2}, \\
& \mathrm{I}_{3}=m^{2} \mathrm{~F}_{3} \text {, } \\
& \mathrm{I}_{4}=-s \mathrm{~F}_{4} \text {, } \\
& \mathrm{I}_{5}=\left(m^{2}-s\right)\left(2 \mathrm{~F}_{4}+\mathrm{F}_{5}\right), \\
& \mathrm{I}_{6}=u \mathrm{~F}_{6} \text {, } \\
& \mathrm{I}_{7}=-2 m^{2} \mathrm{~F}_{6}+\left(u-m^{2}\right) \mathrm{F}_{7} \text {, } \\
& \mathrm{I}_{8}=-t \mathrm{~F}_{8} \text {, } \\
& \mathrm{I}_{9}=\left(m^{2}-s\right) \mathrm{F}_{9}, \\
& \mathrm{I}_{10}=\lambda_{t} \mathrm{~F}_{10} \text {, } \\
& \mathrm{I}_{11}=\left(u-m^{2}\right) \mathrm{F}_{11} \text {, } \\
& \mathrm{I}_{12}=m^{2}\left(u-m^{2}\right) \mathrm{F}_{12} \text {, } \\
& \mathrm{I}_{13}=\left(m^{2}-s\right) \mathrm{F}_{13}, \\
& \mathrm{I}_{14}=m^{2}\left(m^{2}-s\right) \mathrm{F}_{14}, \\
& \mathrm{I}_{15}=\left(m^{2}-s\right) \mathrm{F}_{15} \text {, } \\
& \mathrm{I}_{16}=m^{2}\left(m^{2}-s\right) \mathrm{F}_{16} \text {, } \\
& \mathrm{I}_{17}=3 m^{2} \mathrm{~F}_{15}+2 m^{4} \mathrm{~F}_{16}+m^{2}\left(2 m^{2}-s\right) \mathrm{F}_{17}, \quad \mathrm{I}_{18}=\left(m^{2}-s\right) \mathrm{F}_{18}, \\
& \mathrm{I}_{19}=m^{2}\left(m^{2}-s\right) \mathrm{F}_{19} \text {, } \\
& \mathrm{I}_{20}=\lambda_{t} \mathrm{~F}_{20} \text {, } \\
& \mathrm{I}_{21}=\left(\lambda_{t}-t\right)\left(\frac{1}{2} \mathrm{~F}_{10}-2 \mathrm{~F}_{20}\right)-m^{2} t \mathrm{~F}_{21} \text {, } \\
& \mathrm{I}_{22}=-\left(m^{2}-s\right) t \mathrm{~F}_{22} \text {, } \\
& \mathrm{I}_{23}=\left(u-m^{2}\right) \mathrm{F}_{23}, \\
& \mathrm{I}_{24}=-m^{2} t \mathrm{~F}_{24} \text {, } \\
& \mathrm{I}_{25}=\left(m^{2}-s\right) \mathrm{F}_{25} \text {, }
\end{aligned}
$$




$$
\begin{aligned}
& \mathrm{I}_{26}=-m^{2} t \mathrm{~F}_{26}, \\
& \mathrm{I}_{27}=\left(m^{2}-s\right) u \mathrm{~F}_{27}, \\
& \mathrm{I}_{28}=-m^{2}\left(m^{2}-s\right)\left(\mathrm{F}_{27}-\left(u-m^{2}\right) \mathrm{F}_{28}\right), \quad \mathrm{I}_{29}=-t \mathrm{~F}_{29}, \\
& \mathrm{I}_{30}=\left(m^{2}-s\right)\left(u-m^{2}\right) \mathrm{F}_{30}, \quad \mathrm{I}_{31}=\lambda_{t} \mathrm{~F}_{31}, \\
& \mathrm{I}_{32}=m^{2}\left(m^{2}-s\right) \mathrm{F}_{32}, \quad \mathrm{I}_{33}=\left(m^{2}-s\right)\left(u-m^{2}\right) \mathrm{F}_{33}, \\
& \mathrm{I}_{34}=\frac{1}{6}\left(u-m^{2}\right)\left(2 \mathrm{~F}_{4}+\mathrm{F}_{5}-12 \mathrm{~F}_{13}-12 m^{2} \mathrm{~F}_{14}+6 \mathrm{~F}_{34}\right)+\left(u-m^{2}\right)^{2} \mathrm{~F}_{33}, \\
& \mathrm{I}_{35}=\left(m^{2}-s\right) \lambda_{t} \mathrm{~F}_{35}, \quad \mathrm{I}_{36}=\left(m^{2}-s\right)\left(2 F_{35}\left(t-\lambda_{t}\right)+2 F_{23}+F_{36}\right), \\
& \mathrm{I}_{37}=\sqrt{m^{2}} \sqrt{m^{2}-s} \sqrt{u-m^{2}} \sqrt{-t} \mathrm{~F}_{37}, \quad \mathrm{I}_{38}=-\left(m^{2}-s\right)\left(\mathrm{F}_{23}-\mathrm{F}_{38}\right), \\
& \mathrm{I}_{39}=\left\{\frac{1}{4}\left[\mathrm{~F}_{7}-4\left(m^{2} \mathrm{~F}_{12}+\mathrm{F}_{11}+\mathrm{F}_{20}-\mathrm{F}_{31}+\mathrm{F}_{40}\right)\right]\left(u-m^{2}+\lambda_{t}\right)-\mathrm{F}_{25}+\mathrm{F}_{39}\right\} \\
& +m^{2} \mathrm{~F}_{3}\left(1+\frac{\lambda_{t}}{u-m^{2}}\right)-\left(m^{2}-s\right) \lambda_{t} \mathrm{~F}_{37}+\frac{1}{2} \mathrm{~F}_{6}\left[u-\left(u+m^{2}\right)\left(1+\frac{\lambda_{t}}{u-m^{2}}\right)\right], \\
& \mathrm{I}_{40}=-m^{2} \mathrm{~F}_{3}\left(1+\frac{\lambda_{t}}{u-m^{2}}\right)-\frac{1}{4}\left[\mathrm{~F}_{7}-4\left(m^{2} \mathrm{~F}_{12}+\mathrm{F}_{11}\right)\right]\left(u-m^{2}+\lambda_{t}\right) \\
& +\left(m^{2}-s\right) \lambda_{t} \mathrm{~F}_{37}+\lambda_{t} \mathrm{~F}_{40}+\frac{\mathrm{F}_{6}}{2}\left(u+m^{2}\right)\left(1+\frac{\lambda_{t}}{u-m^{2}}\right), \\
& \mathrm{I}_{41}=\left(m^{2}-s\right)^{2} \mathrm{~F}_{41}, \quad \mathrm{I}_{42}=\left(m^{2}-s\right)\left(u-m^{2}\right) \mathrm{F}_{42}, \\
& \mathrm{I}_{43}=-\left(m^{2}-s\right) t \mathrm{~F}_{43}, \quad \mathrm{I}_{44}=\left(m^{2}-s\right) \mathrm{F}_{44},
\end{aligned}
$$

where we introduced the abbreviation $\lambda_{t}=\sqrt{-t} \sqrt{4 m^{2}-t}$.

By combining the two DEQs in $w$ and $z$ into a single total differential, we get

$$
d \mathbf{I}=\epsilon d \mathbb{A} \mathbf{I}
$$

where $\mathbf{I}$ is a vector that collects the 44 MIs and

$$
d \mathbb{A}=\sum_{i=1}^{12} \mathbb{M}_{i} d \log \left(\eta_{i}\right),
$$

with the $\mathbb{M}_{i}$ being constant matrices with rational entries. The arguments $\eta_{i}$ of this $d$ logform, which define the so-called alphabet of the DEQs, are the following 12 letters:

$$
\begin{aligned}
\eta_{1} & =w, & \eta_{2} & =1+w, \\
\eta_{3} & =1-w, & \eta_{4} & =z, \\
\eta_{5} & =1+z, & \eta_{6} & =1-z, \\
\eta_{7} & =w+z, & \eta_{8} & =z-w, \\
\eta_{9} & =z^{2}-w, & \eta_{10} & =1-w+w^{2}-z^{2}, \\
\eta_{11} & =1-3 w+w^{2}+z^{2}, & \eta_{12} & =z^{2}-w^{2}-w z^{2}+w^{2} z^{2} .
\end{aligned}
$$

In the present work, we compute the MIs in the kinematic region where all the letters are real and positive,

$$
0<w<1 \wedge \sqrt{w}<z<\sqrt{1-w+w^{2}}
$$




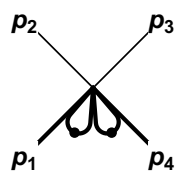

$\mathcal{T}_{1}$

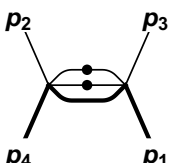

$\mathcal{T}_{7}$

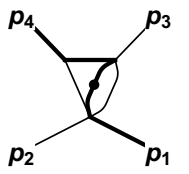

$\mathcal{T}_{13}$

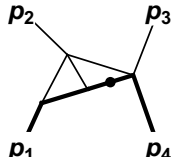

$\mathcal{T}_{19}$

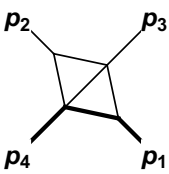

$\mathcal{T}_{25}$

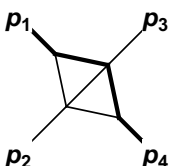

$\mathcal{T}_{31}$

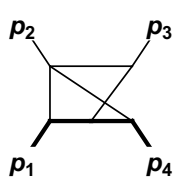

$\mathcal{T}_{37}$

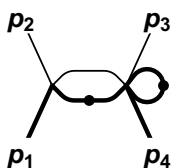

$\mathcal{T}_{2}$

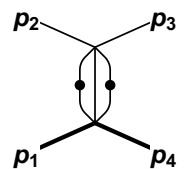

$\mathcal{T}_{8}$

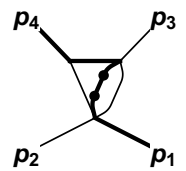

$\mathcal{T}_{14}$

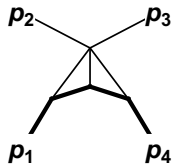

$\mathcal{T}_{20}$

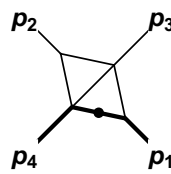

$\mathcal{T}_{26}$

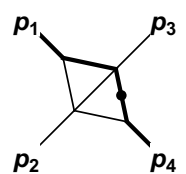

$\mathcal{T}_{32}$

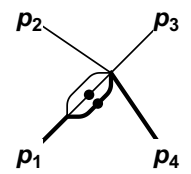

$\mathcal{T}_{3}$

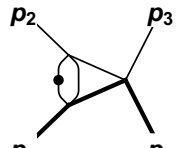

$\mathcal{T}_{9}$

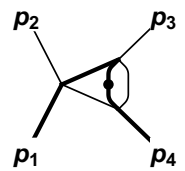

$\mathcal{T}_{15}$

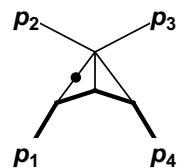

$\mathcal{T}_{21}$

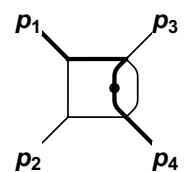

$\mathcal{T}_{27}$

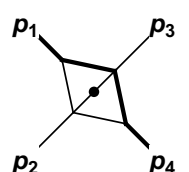

$\mathcal{T}_{33}$

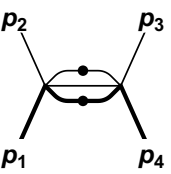

$\mathcal{T}_{4}$

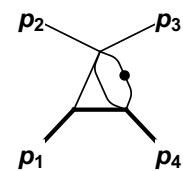

$\mathcal{T}_{10}$

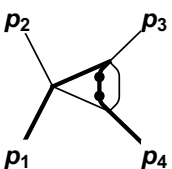

$\mathcal{T}_{16}$

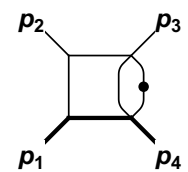

$\mathcal{T}_{22}$

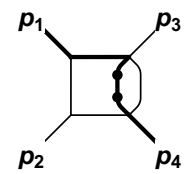

$\mathcal{T}_{28}$

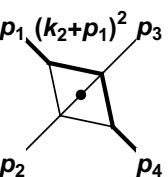

$\mathcal{T}_{34}$

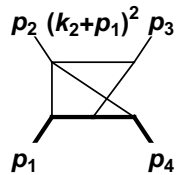

$\mathcal{T}_{38}$

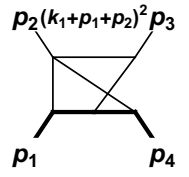

$\mathcal{T}_{39}$

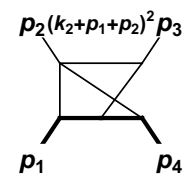

$\mathcal{T}_{40}$

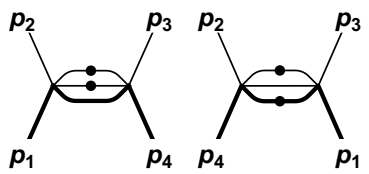

$\mathcal{T}_{5}$

$\mathcal{T}_{6}$

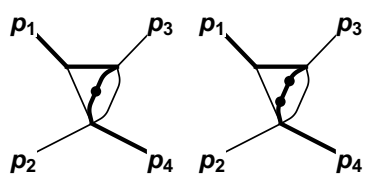

$\mathcal{T}_{11}$

$\mathcal{T}_{12}$
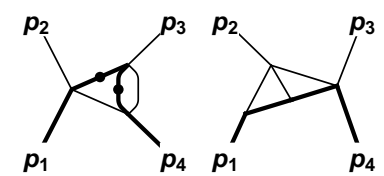

$\mathcal{T}_{17}$

$\mathcal{T}_{18}$

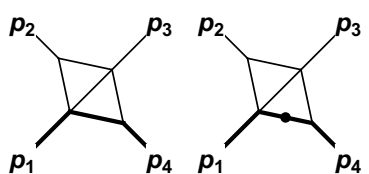

$\mathcal{T}_{23}$

$\mathcal{T}_{24}$
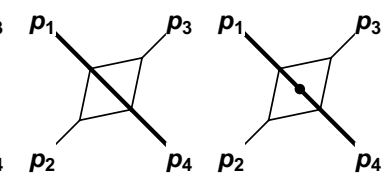

$\mathcal{T}_{29}$

$\mathcal{T}_{30}$
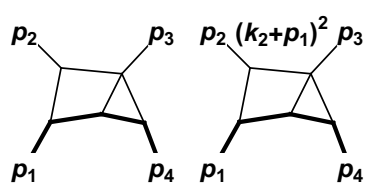

$\mathcal{T}_{35}$

$\mathcal{T}_{36}$
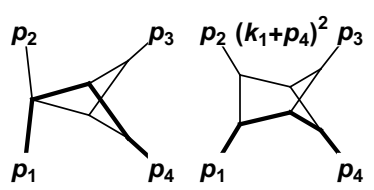

$\mathcal{T}_{42}$

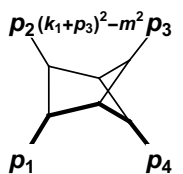

$\mathcal{T}_{43}$

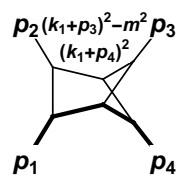

$\mathcal{T}_{44}$

Figure 2. The 44 MIs $\mathcal{T}_{1, \ldots, 44}$ for the two-loop non-planar topology $T_{6}$. Thin lines represent massless propagators and thick lines stand for massive ones. Each dot indicates an additional power of the corresponding propagator. Numerator insertions are indicated explicitly on top of each diagram. 
which corresponds to the unphysical region

$$
t<0 \wedge s<0 .
$$

The analytic continuation to the physical region for $\mu e$ scattering

$$
s \geq m^{2} \wedge-s\left(1-\frac{m^{2}}{s}\right)^{2} \leq t \leq 0 \wedge 2 m^{2}-s \leq u \leq \frac{m^{4}}{s},
$$

can be obtained through the Feynman prescription, by adding a small positive imaginary part $i \omega$ to the Mandelstam invariants whenever they become positive.

Since all the integrals defined in eq. (3.3) are finite in the $\epsilon \rightarrow 0$ limit, the vector $\mathbf{I}(\epsilon, w, z)$ admits a Taylor expansion in $\epsilon$ (of uniform transcendental weight),

$$
\mathbf{I}(\epsilon, w, z)=\mathbf{I}^{(0)}(w, z)+\epsilon \mathbf{I}^{(1)}(w, z)+\epsilon^{2} \mathbf{I}^{(2)}(w, z)+\ldots,
$$

with the $n$-th order coefficient given by

$$
\mathbf{I}^{(n)}(w, z)=\sum_{i=0}^{n} \Delta^{(n-i)}\left(w, z ; w_{0}, z_{0}\right) \mathbf{I}^{(i)}\left(w_{0}, z_{0}\right),
$$

where $\mathbf{I}^{(i)}\left(w_{0}, z_{0}\right)$ is a constant vector and $\Delta^{(k)}$ the weight- $k$ operator

$$
\Delta^{(k)}\left(w, z ; w_{0}, z_{0}\right)=\int_{\gamma} \underbrace{d \mathbb{A} \ldots d \mathbb{A}}_{\mathrm{k} \text { times }}, \quad \Delta^{(0)}\left(w, z ; w_{0}, z_{0}\right)=1,
$$

that iterates $k$ ordered integrations of the 1-form $d \mathbb{A}$ along any piecewise-smooth path $\gamma:[0,1] \rightarrow M \subset \mathbb{C} \times \mathbb{C}$ such that $\gamma(0)=\left(w_{0}, z_{0}\right)$ and $\gamma(1)=(w, z)$. If the singularities of the integrand are removed from $M$, and suitable branch cuts are imposed, the iterated integrals in eq. (3.12) do not depend on the chosen path (see e.g. ref. [21] for a compact review of the properties of the iterated path integrals). Since the rational alphabet given in eq. (3.6) has only algebraic roots, we can directly express (by first integrating in $w$ and then in $z$ or viceversa ${ }^{3}$ ) the iterated integrals of eq. (3.12) in terms of GPLs, which are defined as

$$
\begin{aligned}
& G\left(\vec{a}_{n} ; x\right) \equiv G\left(a_{1}, \vec{a}_{n-1} ; x\right) \equiv \int_{0}^{x} d t \frac{1}{t-a_{1}} G\left(\vec{a}_{n-1} ; t\right), \\
& G\left(\overrightarrow{0}_{n} ; x\right) \equiv \frac{1}{n !} \log ^{n}(x) .
\end{aligned}
$$

The length $n$ of the vector $\vec{a}_{n}$ corresponds the transcendental weight of $G\left(\vec{w}_{n} ; x\right)$ and it amounts to the number of iterated integrations that define the GPL. The GPLs in our

\footnotetext{
${ }^{3}$ The integration to GPLs of a rational $d \log$ form, for instance first in $w$ and then in $z$, can be performed in two ways. First, one can use the iterated path-integral approach and choose $\gamma$ to be a sequence of straight lines, from $\left(w_{0}, z_{0}\right)$ to $\left(w, z_{0}\right)$ and then to $(w, z)$. Equivalently (see e.g. ref. [19]) one can work directly on the associated canonical partial DEQs, $\partial_{i} \mathbf{I}=\epsilon \partial_{i} \mathbb{A} \mathbf{I}$ order by order in $\epsilon(i=w, z)$. In particular, one first integrates the partial DEQ in $w$ up to an unknown vector $\mathbf{H}$ depending on $z$. An ordinary DEQ for $\mathbf{H}$ is then obtained by explicitly taking the derivative of the integral of the partial DEQ in $w$ and matching to the partial DEQ in $z$. For definiteness, the latter is the strategy we followed to produce the results of this work.
} 
solution, which for definiteness we obtain by first integrating in $w$ and then in $z$, are of two classes, namely GPLs in $w$, with weights drawn from the set

$$
\left\{0, \pm 1, \pm z, z^{2}, \frac{1}{2}\left(3 \pm \sqrt{5-4 z^{2}}\right), \frac{z\left(z \pm \sqrt{4-3 z^{2}}\right)}{2\left(z^{2}-1\right)}, \frac{1}{2}\left(1 \pm \sqrt{4 z^{2}-3}\right)\right\}
$$

and GPLs in $z$, with weights drawn from

$$
\{0, \pm 1, \pm i\}
$$

The analytic structure of the canonical MIs is determined by the letters at all orders in $\epsilon$, and the solution can in principle be built up to any weight. We compute the MIs up to weight 4 , which will be enough for the two-loop virtual calculation.

In the region defined by eq. (3.7), the imaginary part of our solution $\mathbf{I}(\epsilon, w, z)$ only originates from the integration constants $\mathbf{I}^{(i)}\left(w_{0}, z_{0}\right)$.

\subsection{Boundary conditions}

The general solution of the system of DEQs in terms of GPLs, which is obtained from the integration of eq. (3.4), must be complemented by a suitable set of boundary conditions. These boundary conditions can be determined either from the knowledge of the analytic expression of the MIs in special kinematic configurations or by imposing their regularity at pseudo-thresholds of the DEQs. For the problem under consideration, regularity conditions express the boundary constant as combinations of GPLs of argument 1, with weights drawn from the set $a_{i} \in\{-1,-i, 0, i, 1\}$, which arises from the kinematic limits imposed on the alphabet given in eq. (3.6). We used GiNaC to numerically verify that for each MI, at each order in $\epsilon$, the corresponding combination of constant GPLs is proportional to a uniform combination of the transcendental constants $\pi, \zeta_{k}$ and $\log 2$.

In the following, we specify how the boundary constants of each integral have been obtained:

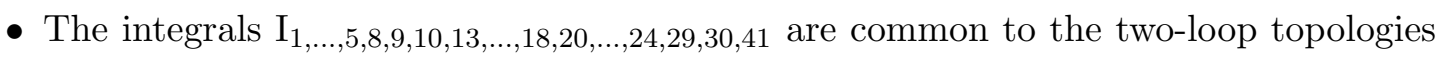
discussed in ref [1], to which we refer the reader for the discussion of the boundary fixing. Furthermore, the integrals $\mathrm{I}_{25,26}$ are related to $\mathrm{I}_{23,24}$ by $s \leftrightarrow u$ crossing, so that their boundary constants can be inferred directly from the ones of $\mathrm{I}_{23,24}$.

- The integrals $\mathrm{I}_{6,7}$ are regular in the limit $u \rightarrow 0$, where they can be reduced, via IBPs, to a single two-loop vacuum diagram. From the analytic expression of the latter, we obtain the boundary values

$$
\begin{aligned}
& \left.\mathrm{I}_{6}\right|_{u=0}=0 \\
& \left.\mathrm{I}_{7}\right|_{u=0}=1+\frac{\pi^{2}}{3} \epsilon^{2}-2 \zeta_{3} \epsilon^{3}+\frac{\pi^{4}}{10} \epsilon^{4}+\mathcal{O}\left(\epsilon^{5}\right) .
\end{aligned}
$$


- The integrals $\mathrm{I}_{11,12}$ are regular in the limit $u \rightarrow 0$. In particular, we observe that their boundary values can be obtained as the limits

$$
\left.\mathrm{I}_{11}\right|_{u=0}=-\epsilon^{3} m^{2} \lim _{p_{1}^{2} \rightarrow m^{2}}
$$

Therefore, we can generate the DEQs for the analogous triangle integrals with $p_{4}^{2}=0$, and $u=0$ and an off-shell leg $p_{1}^{2}$, solve them by using as an integration base-point the regular point $p_{1}^{2}=0$, and finally extract the boundary values of $\mathrm{I}_{11}$ and $\mathrm{I}_{12}$ by means of eq. (3.18). The details of this computation are reported in appendix A. In this way, we obtain

$$
\begin{aligned}
& \left.\mathrm{I}_{11}\right|_{u=0}=2 \zeta_{3} \epsilon^{3}+\frac{7 \pi^{4}}{180} \epsilon^{4}+\mathcal{O}\left(\epsilon^{5}\right), \\
& \left.\mathrm{I}_{12}\right|_{u=0}=-\frac{\pi^{2}}{12} \epsilon^{2}-\frac{5 \zeta_{3}}{2} \epsilon^{3}-\frac{1}{12} \pi^{4} \epsilon^{4}+\mathcal{O}\left(\epsilon^{5}\right) .
\end{aligned}
$$

- The boundary constants of the integrals $\mathrm{I}_{27,28}$ can be fixed by imposing the regularity of their DEQs as $t \rightarrow 0$.

- The boundary constants of the integrals $\mathrm{I}_{31,32,33}$ are obtained by demanding regularity at $t \rightarrow 0$, as well the reality of the integrals in the region $s \leq 0, u \leq 0$.

- The boundary constants of the integrals $\mathrm{I}_{19,34,35 \ldots 40,42,43,44}$ are obtained by demanding their finiteness in the limit $s \rightarrow \frac{\sqrt{4 m^{2}-t}-\sqrt{-t}}{\sqrt{4 m^{2}-t}+\sqrt{-t}}$.

The analytic expressions of the MIs are given in electronic form in the ancillary files attached to the arXiv version of the manuscript.

\section{Numerical evaluation of the non-planar four point integrals}

The analytic expression of our MIs have been numerically evaluated in the region $s, t<0$ by means of the GiNac library, and successfully checked against independent calculations. In particular, the integrals $I_{i}$ with $i=1, \ldots, 36,41$ were computed with the package SecDec. For the most complex topologies, corresponding to the non-planar four-point integrals $I_{i}$ with $i=37, \ldots, 40,42,43,44$, we adopted a different strategy. As the numerical evaluation of those integrals is challenging, we identified an alternative set of independent MIs that are quasi finite [29] in $d=6$. The latter have been computed semi-numerically by means of an in-house algorithm: starting from the Feynman parametrisation of the integrals, we carried out as many analytic integration as possible, until we reached a form where the left over multivariate integral could be numerically evaluated by means of Gauss quadrature. Dimension-shifting identities [46, 47] and IBPs, implemented in LiteRed [48, 49], establish analytical relations between this set of integrals and the MIs we computed around $d=4$. 


\begin{tabular}{|c|c|c|}
\hline graph & $I^{[d]}$-integral & $I^{[d=6-2 \epsilon]}\left(s=-\frac{1}{7}, t=-\frac{1}{3}, m^{2}=1\right)$ \\
\hline & $I^{[d]}(1,1,1,0,1,0,1,1,1)$ & $-1.219372-i 0.294408$ \\
\hline & $I^{[d]}(1,2,1,0,1,0,1,1,1)$ & $0.98317+i 1.00335$ \\
\hline & $I^{[d]}(1,1,1,0,1,0,1,2,1)$ & $12.039969+i 6.660946$ \\
\hline & $I^{[d]}(1,1,1,0,0,0,1,1,1)$ & $\frac{1}{4 \epsilon}+0.6798187+i 0.0300909$ \\
\hline & $I^{[d]}(2,1,1,0,0,0,1,1,1)$ & $-0.554605-i 0.06984485$ \\
\hline & $I^{[d]}(1,1,1,0,0,0,1,2,1)$ & $-1.91103+i 0.241649$ \\
\hline & $I^{[d]}(3,1,1,0,0,0,1,1,1)$ & $0.525679+i 0.248668$ \\
\hline
\end{tabular}

Table 1. Numerical results for our set of quasi-finite non-planar MIs belonging to the 6- and 7-denominators topologies $\left(m^{2}=1\right)$.

The definition of the 7 non-planar MIs that are quasi finite in $d=6$ dimensions, together with our results at the phase-space point $s=-1 / 7, t=-1 / 3, m^{2}=1$, are collected in table 1 . We identified them through educated guesses or with the help of Reduze. In the next subsection, we use the first of those integrals as an example to describe our evaluation strategy.

\subsection{The non-planar box in $d=6$ dimensions}

As an example, we describe the numerical evaluation of the non-planar scalar integral

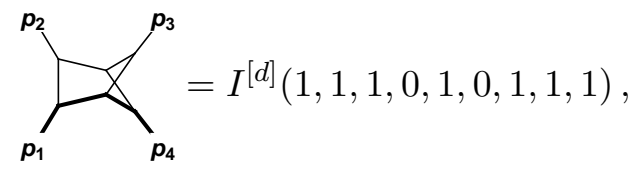

carried out in two steps. 


\subsubsection{Analytic integrations}

By using Feynman parametrisation, the integral can be written as

$$
\underbrace{p_{2}}_{p_{1}}=\Gamma(7) \int \widetilde{\mathrm{d}^{d} k_{1}} \widetilde{\mathrm{d}^{d} k_{2}} \int_{0}^{1} d x_{1} \ldots \int_{0}^{1} d x_{7} \frac{\delta\left(1-x_{1234567}\right)}{D_{\text {tot }}^{7}},
$$

where

$$
D_{\text {tot }}=x_{1} D_{1}+x_{2} D_{2}+x_{3} D_{3}+x_{4} D_{5}+x_{5} D_{7}+x_{6} D_{8}+x_{7} D_{9}+i \omega .
$$

After integrating over $k_{1}$ and $k_{2}$, one finds

$$
\begin{aligned}
\Gamma_{\epsilon}^{2} \overbrace{\boldsymbol{p}_{1}}^{\boldsymbol{p}_{2}}= & -\Gamma(7-d) \int_{0}^{1} d x_{1} \ldots \int_{0}^{1} d x_{7} \frac{\delta\left(1-x_{1234567}\right)}{A_{0}^{\frac{3 d}{2}-7} \Delta^{7-d}}, \\
A_{0}= & x_{34} x_{56}+x_{5} x_{6}+x_{346} x_{7}+x_{2} x_{3457}+x_{1} x_{2567}, \\
\Delta= & x_{2}^{2} x_{3457}+x_{1}^{2} x_{2567}+x_{1} x_{2}\left(x_{2}+2 x_{57}\right)+x_{3}\left(-x_{5} x_{6}+x_{2} x_{7}\right) t \\
& +\left(x_{2}\left(-x_{4} x_{5}+x_{3} x_{7}\right)-x_{1}\left(x_{4} x_{256}+x_{46} x_{7}\right)\right)(s-1)-i \omega A_{0},
\end{aligned}
$$

where we used the notation $x_{i_{1} i_{2} \ldots i_{n}}=x_{i_{1}}+x_{i_{2}}+\ldots+x_{i_{n}}$. We perform as many analytic integrations as possible. In particular, we integrate over $x_{3}$ eliminating the $\delta$-function, and we make the changes of variables $x_{6} \rightarrow x_{26}-x_{2}, x_{7} \rightarrow x_{57}-x_{5}$. In this way, the polynomial $\Delta$ becomes linear in $x_{4}$ and $x_{5}$, so that eq. (4.4) becomes

$$
\begin{aligned}
& \Gamma_{\epsilon}^{2} \underbrace{\boldsymbol{p}_{2}}_{\boldsymbol{p}_{1}}=-\Gamma(7-d) \int_{0}^{\boldsymbol{p}_{3}} d x_{26} \int_{0}^{1-x_{26}} \frac{d x_{57}}{A^{\frac{3 D}{2}-7}} \int_{0}^{1-x_{2657}} d x_{1} \int_{0}^{x_{26}} d x_{2} \int_{0}^{x_{57}} d x_{5} \times \\
& \frac{d x_{4}}{\left(C_{41} x_{4}+C_{40}\right)^{7-d}},
\end{aligned}
$$

where

$$
\begin{aligned}
A= & x_{2657}\left(1-x_{2657}\right)+x_{26} x_{57}, \\
C_{41}= & t x_{26} x_{5}-(s+t-1) x_{2} x_{57}-(s-1) x_{1} x_{2657}, \\
C_{40}= & x_{1}^{2} x_{2657}+x_{2}\left(x_{2}\left(1-x_{26}\right)+2 x_{1} x_{57}\right)+t\left(-1+x_{12657}\right)\left(x_{26} x_{5}-x_{2} x_{57}\right)-i \omega A \\
& +(s-1)\left(x_{5}-x_{57}\right)\left(x_{1} x_{26}+x_{2}\left(-1+x_{2657}\right)\right) .
\end{aligned}
$$

The integral over $x_{4}$ in eq. (4.6) is finite for $d \rightarrow 6$ and, in this limit, we get

$$
\begin{aligned}
& \Gamma_{\epsilon}^{2} \overbrace{p_{1}}^{p_{2}} \stackrel{d \rightarrow 6}{=}-\int_{0}^{p_{3}} d x_{26} \int_{0}^{1-x_{26}} \frac{d x_{57}}{A^{2}} \int_{0}^{1-x_{2657}} d x_{1} \int_{0}^{x_{26}} d x_{2} \times \\
& \int_{0}^{x_{57}} \frac{d x_{5}}{x_{5} t x_{26}-f_{3}} \ln \left(\frac{f_{4} x_{5}+P_{4}}{f_{2} x_{5}+P_{3}}\right),
\end{aligned}
$$


where

$$
\begin{aligned}
& P_{1}=x_{2}^{2}\left(1-x_{26}\right)+2 x_{1} x_{2} x_{57}+x_{1}^{2} x_{2657}+t x_{57}\left(x_{26}-x_{2}\right)\left(-1+x_{12657}\right)-i \omega A, \\
& P_{2}=x_{2}^{2}\left(1-x_{26}\right)+2 x_{1} x_{2} x_{57}+x_{1}^{2} x_{2657}+(s-1)\left(-1+x_{12657}\right)\left(x_{1} x_{2657}+x_{2} x_{57}\right)-i \omega A, \\
& P_{3}=P_{1}-f_{2} x_{57}, \\
& P_{4}=P_{2}-f_{4} x_{57},
\end{aligned}
$$

and

$$
\begin{aligned}
& f_{1}=f_{3}-t x_{26} x_{57} \\
& f_{2}=f_{4}-t x_{26}\left(1-x_{12657}\right) \\
& f_{3}=(s+t-1) x_{2} x_{57}+(s-1) x_{1} x_{2657} \\
& f_{4}=(s-1)\left(x_{1} x_{26}+x_{2}\left(-1+x_{2657}\right)\right)
\end{aligned}
$$

Finally, we integrate over $x_{5}$, and reduce eq. (4.8) to

$$
\begin{array}{r}
\Gamma_{\epsilon}^{2} \overbrace{p_{1}}^{p_{2}} \stackrel{d \rightarrow 6}{=}-\int_{0}^{1} \frac{d x_{26}}{t x_{26}} \int_{0}^{1-x_{26}} \frac{d x_{57}}{A^{2}} \int_{0}^{1-x_{2657}} d x_{1} \int_{0}^{x_{26}} d x_{2} \times \\
\left(\operatorname{Li}_{2}\left(\frac{Q_{1}}{R}\right)-\operatorname{Li}_{2}\left(\frac{Q_{2}}{R}\right)-\operatorname{Li}_{2}\left(\frac{Q_{3}}{R}\right)+\operatorname{Li}_{2}\left(\frac{Q_{4}}{R}\right)\right),
\end{array}
$$

where

$$
\begin{aligned}
Q_{1} & =f_{1} f_{2}, \quad Q_{2}=f_{1} f_{4}, \quad Q_{3}=f_{3} f_{2}, \quad Q_{4}=f_{3} f_{4}, \\
R & =Q_{i}+P_{i} t x_{26}, \quad \forall i, i=1, \ldots, 4 .
\end{aligned}
$$

\subsubsection{Numerical integrations}

The four remaining integration variables in eq. (4.11) are rescaled, and mapped onto a four-dimensional hypercube of unit side,

$$
x_{26}=t_{1}, x_{57}=\left(1-x_{26}\right) t_{2}, x_{1}=\left(1-x_{2657}\right) t_{3}, x_{2}=\left(x_{26}\right) t_{4} ;
$$

so that the new variables $t_{i}$ have to be integrated over $[0,1]$. At this point, we have to consider the branch points of the dilogarithms that appear in eq. (4.11), which correspond the hypersurfaces defined by the equations

$$
R\left(t_{1}, t_{2}, t_{3}, t_{4}\right)=0, \quad P_{i}\left(t_{1}, t_{2}, t_{3}, t_{4}\right)=0, \quad i=1, \ldots 4 .
$$

It is necessary to sample carefully the integrand near these branch points. Therefore, for the integration over $t_{4}$, we split the integration interval at the $N_{4}\left(t_{1}, t_{2}, t_{3}\right)$ real solutions $z_{4 j}\left(t_{1}, t_{2}, t_{3}\right)$ of eq. (4.14) which are on the interval $[0,1]$,

$$
\int_{0}^{1} d t_{4}=\sum_{j=0}^{N_{4}-1} \int_{z_{4 j}\left(t_{1}, t_{2}, t_{3}\right)}^{z_{4, j+1}\left(t_{1}, t_{2}, t_{3}\right)} d t_{4}, z_{40}=0, z_{4 N_{4}}=1 .
$$


Analogously, for the integration over $t_{3}$, we split the integration interval at the $N_{3}\left(t_{1}, t_{2}\right)$ real zeros $z_{3 j}\left(t_{1}, t_{2}\right)$ of the discriminants (polynomials in $\left(t_{1}, t_{2}, t_{3}\right)$ ) that appear in the zeros $z_{4 j}$. These are the points where the hypersurfaces of eq. (4.14) are tangent to the hyperplane $t_{4}=$ constant,

$$
\int_{0}^{1} d t_{3}=\sum_{j=0}^{N_{3}-1} \int_{z_{3 j}}^{z_{3, j+1}} d t_{3}, \quad z_{30}=0, z_{3 N_{3}}=1
$$

Analogously, for the integration over $t_{2}$, we split the integration interval at the $N_{2}\left(t_{1}\right)$ zeros $z_{2 j}\left(t_{1}\right)$ of the discriminants (polynomials in $\left(t_{1}, t_{2}\right)$ ) that appear in the zeros $z_{3 j}$,

$$
\int_{0}^{1} d t_{2}=\sum_{j=0}^{N_{2}-1} \int_{z_{2 j}}^{z_{2, j+1}} d t_{2}, \quad z_{20}=0, z_{2 N_{2}}=1
$$

We proceed in a similar way for the last integration,

$$
\int_{0}^{1} d t_{1}=\sum_{j=0}^{N_{1}-1} \int_{z_{1 j}}^{z_{1, j+1}} d t_{1}, \quad z_{10}=0, z_{1 N_{1}}=1
$$

To carry out the integration over a generic interval $\left[t_{a}, t_{b}\right]$, we perform the change of variables $t_{i} \rightarrow u_{i}$, with

$$
t_{i}=t_{a i}+\frac{e^{u_{i}^{3}}}{e^{u_{i}^{3}}+1}\left(t_{b i}-t_{a i}\right), \quad i=1, \ldots, 4
$$

in order to deal with possible singularities at the endpoints. The variable $u_{i}$ should be integrated in $(-\infty, \infty)$ but we actually truncate the integration domain to $(-M,+M)$, with $M$ suitably large (typically $M \sim 4$ ), and we use Gauss-Legendre integration over 16 points. Note that all the singularities in the integrands are logarithmic, and therefore integrable, so we can safely set a very small value of $\omega$, like $10^{-30}$.

By using 16 subdivisions in each interval and in every variable we find that our integral, in the phase space point $s=-1 / 7, t=-1 / 3, m^{2}=1$, amounts to

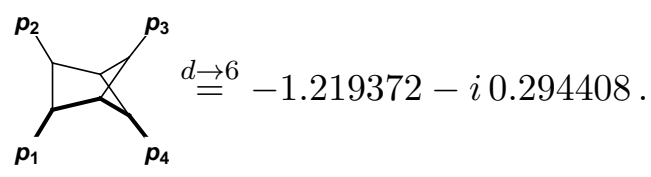

A similar procedure is adopted for the other integrals in table 1. Case-by-case, after the analytic integrations, the corresponding integrands, in the $d \rightarrow 6$ limit, are found to be combinations of logarithms, so that the decomposition of the integration domain, and the numerical integration can be carried out along the same lines as for the non-planar scalar box integral. 


\section{Conclusions}

In this work, we presented the analytic evaluation of the two-loop master integrals needed to compute the non-planar Feynman diagrams contributing to $\mu e$ elastic scattering in QED at NNLO. We adopted the same computational strategy previously applied to planar diagrams, and presented in the companion article [1]. Namely, we employed the method of differential equations and of the Magnus exponential to identify a canonical set of master integrals and we derived boundary conditions either from the regularity requirements at pseudothresholds or from the knowledge of the integrals at special kinematic points, possibly evaluated by means of auxiliary, simpler systems of differential equations. The considered master integrals were expressed as a Taylor series around four space-time dimensions, whose coefficients are written as a combination of generalised polylogarithms. We worked in the massless electron approximation, while keeping full dependence on the muon mass.

The scattering of high-energy muons on atomic electrons has been recently proposed as an ideal framework to determine, in a novel way, the leading hadronic contribution to the anomalous magnetic moment of the muon. The ambitious experimental goal of the MUonE project, namely measuring the differential cross section of the $\mu e \rightarrow \mu e$ process with an accuracy of 10ppm, requires, on the theoretical side, the knowledge of the QED corrections at NNLO. The results of the planar and non-planar master integrals we obtained represent an important step towards the evaluation of the virtual corrections at the required order.

By crossing symmetry, our results are also relevant for muon-pair production at $e^{+} e^{-}$colliders operating well below the $Z$-pole, such as Belle II and VEPP-2000, as well as for the QCD corrections to heavy-quark pair production at hadron colliders. The former application is particularly interesting, as a precise knowledge of the differential cross section in QED could be exploited to constrain non-standard ee $\mu \mu$ interactions via the measurement of a forward-backward asymmetry.

\section{Acknowledgments}

We wish to acknowledge stimulating discussions with all members of the MUonE collaboration, and in particular we wish to thank Giovanni Ossola, Massimo Passera and William J. Torres Bobadilla for our constant interaction and collaboration, and Fedor Ignatov for stimulating discussions. We thank Lorenzo Tancredi for suggesting the use of an alternative set of variables, which simplified the form of the canonical system we originally got at an earlier stage of the project. We also acknowledge discussions with Andreas von Manteuffel. We wish to thank Lance Dixon for interesting feedback on the project. We would like to express a special thanks to the Mainz Institute for Theoretical Physics (MITP) for its hospitality and support during the workshop "The evaluation of the leading hadronic contribution to the muon anomalous magnetic moment". U.S. is supported by the DOE contract DE-AC02-06CH11357. This research was supported in part by the Swiss National Science Foundation (SNF) under contract 200020-175595. 


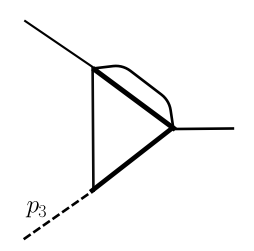

Figure 3. Auxiliary three-point integral family for eq. (3.18). Thick and thin lines represent, respectively, massive and massless propagators. The dashed line corresponds to an external leg with arbitrary squared momentum.

\section{A Evaluation of the auxiliary vertex integrals for eq. (3.18)}

In this appendix, we discuss the solution of the DEQs for the vertex integrals that we used in eq. (3.18) as an input for the determination of the boundary constants of the MIs $\mathrm{I}_{11,12}$.

The required input integrals belong to the integral family

$$
\int \widetilde{\mathrm{d}^{d} k_{1}} \widetilde{\mathrm{d}^{d} k_{2}} \frac{D_{5}^{n_{5}} D_{6}^{n_{6}} D_{7}^{n_{7}}}{D_{1}^{n_{1}} D_{2}^{n_{3}} D_{3}^{n_{3}} D_{4}^{n_{4}}}, \quad n_{i} \geq 0
$$

which is identified by the set of denominators

$$
\begin{aligned}
& D_{1}=k_{1}^{2}, \quad D_{2}=k_{2}^{2}-m^{2}, \quad D_{3}=\left(k_{1}+k_{2}+p_{1}\right)^{2}, \quad D_{4}=\left(k_{1}+p_{1}+p_{2}\right)^{2}-m^{2}, \\
& D_{5}=\left(k_{1}+p_{2}\right)^{2}, \quad D_{6}=\left(k_{2}+p_{1}\right)^{2}, \quad D_{7}=\left(k_{2}+p_{2}\right)^{2},
\end{aligned}
$$

and by the external momenta

$$
p_{1}^{2}=p_{2}^{2}=0, \quad p_{3}^{2}=\left(p_{1}+p_{2}\right)^{2} .
$$

A representative 4-propagator integral of this family is depicted in figure 3. IBPs reduce the integral family of eq. (A.1) to a set of 5 MIs, whose dependence on $p_{3}^{2}$ is parametrised in terms of the dimensionless variable

$$
x=-\frac{p_{3}^{2}}{m^{2}}
$$

The integral basis

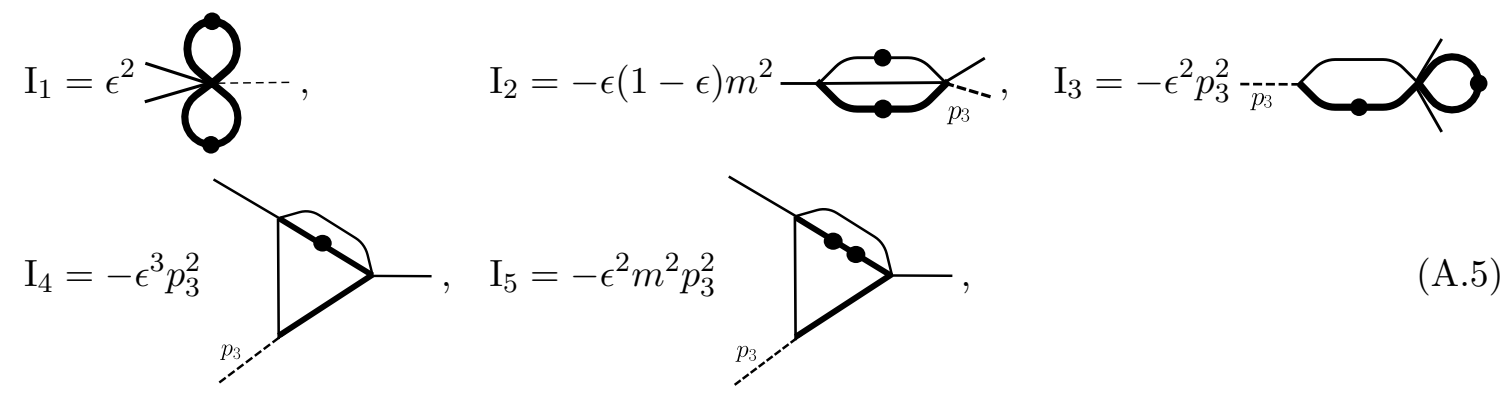

fulfils canonical DEQs,

$$
d \mathbf{I}=\epsilon d \mathbb{A} \mathbf{I}
$$


where

$$
d \mathbb{A}=\mathbb{M}_{0} d \log x+\mathbb{M}_{-1} d \log (x+1)
$$

with

$$
\mathbb{M}_{0}=\left(\begin{array}{ccccc}
0 & 0 & 0 & 0 & 0 \\
0 & 0 & 0 & 0 & 0 \\
0 & 0 & 1 & 0 & 0 \\
0 & 0 & 0 & 1 & 0 \\
0 & 0 & -\frac{1}{2} & -3 & -2
\end{array}\right), \quad \mathbb{M}_{1}=\left(\begin{array}{ccccc}
0 & 0 & 0 & 0 & 0 \\
0 & 0 & 0 & 0 & 0 \\
-1 & 0 & -2 & 0 & 0 \\
\frac{1}{2} & -\frac{1}{2} & 0 & -3 & -2 \\
-\frac{1}{2} & \frac{1}{2} & 0 & 3 & 2
\end{array}\right)
$$

The general solution of the DEQs can be expressed in terms of harmonic polylogarithms (HPLs), i.e. GPLs with weights $a_{i} \in\{-1,0,1\}$. The integrals $\mathrm{I}_{1,2}$, which are independent of $x$, are determined by direct integration,

$$
\mathrm{I}_{1}=1, \quad \mathrm{I}_{2}(\epsilon)=1+\frac{\pi^{2}}{3} \epsilon^{2}-2 \zeta_{3} \epsilon^{3}+\frac{\pi^{4}}{10} \epsilon^{4}+\mathcal{O}\left(\epsilon^{5}\right),
$$

whereas the boundary constants of $\mathrm{I}_{3,4,5}$ are obtained by demanding their vanishing in the regular limit $x \rightarrow 0$. In particular, for the two triangle integrals, we obtain

$$
\begin{aligned}
\mathrm{I}_{4}(\epsilon, x)= & \left(\frac{\pi^{2}}{6} G(-1 ; x)-G(-1 ; x) G(0,-1 ; x)+2 G(0,-1,-1 ; x)\right) \epsilon^{3} \\
& +\left(\zeta_{3} G(-1 ; x)+\frac{\pi^{2}}{12} G^{2}(-1 ; x)-\frac{\pi^{2}}{6} G(0,-1 ; x)+\frac{1}{2} G^{2}(-1 ; x) G(0,-1 ; x)\right. \\
& +G(-1 ; x) G(0,0,-1 ; x)-3 G(0,-1,-1,-1 ; x)+2 G(0,-1,0,-1 ; x) \\
& -2 G(0,0,-1,-1 ; x)) \epsilon^{4}+\mathcal{O}\left(\epsilon^{5}\right) \\
\mathrm{I}_{5}(\epsilon, x)= & \frac{1}{2} G(0,-1 ; x) \epsilon^{2}+\left(\frac{\pi^{2}}{6} G(-1 ; x)+G(-1 ; x) G(0,-1 ; x)-3 G(0,-1,-1 ; x)\right. \\
& \left.-\frac{1}{2} G(0,0,-1 ; x)\right) \epsilon^{3}+\left(-\zeta_{3} G(-1 ; x)-\frac{\pi^{2}}{12} G^{2}(-1 ; x)+\frac{\pi^{2}}{6} G(0,-1 ; x)\right. \\
& -\frac{1}{2} G^{2}(-1 ; x) G(0,-1 ; x)-G(-1, x) G(0,0,-1 ; x)+5 G(0,-1,-1,-1 ; x) \\
& \left.+G(0,-1,0,-1 ; x)+3 G(0,0,-1,-1 ; x)+\frac{3}{2} G(0,0,0,-1 ; x)\right) \epsilon^{4}+\mathcal{O}\left(\epsilon^{5}\right) .
\end{aligned}
$$

These solutions are real valued in the interval $0<x<1$. By analytic continuation to the region $x<0$, we can extract the values of the two integral at $p_{3}^{2}=m^{2}$,

$$
\begin{aligned}
& \mathrm{I}_{4}(\epsilon,-1)=2 \zeta_{3} \epsilon^{3}+\frac{7 \pi^{4}}{180} \epsilon^{4}+\mathcal{O}\left(\epsilon^{5}\right), \\
& \mathrm{I}_{5}(\epsilon,-1)=-\frac{\pi^{2}}{12} \epsilon^{2}-\frac{5 \zeta_{3}}{2} \epsilon^{3}-\frac{1}{12} \pi^{4} \epsilon^{4}+\mathcal{O}\left(\epsilon^{5}\right),
\end{aligned}
$$

which can then be used in eq. (3.18). 


\section{B $d \log$ forms}

In this appendix we collect the coefficient matrices of the $d$ log-form

$$
\begin{aligned}
d \mathbb{A}= & \mathbb{M}_{1} d \log (w)+\mathbb{M}_{2} d \log (1+w)+\mathbb{M}_{3} d \log (1-w) \\
& +\mathbb{M}_{4} d \log (z)+\mathbb{M}_{5} d \log (1+z)+\mathbb{M}_{6} d \log (1-z) \\
& +\mathbb{M}_{7} d \log (w+z)+\mathbb{M}_{8} d \log (z-w) \\
& +\mathbb{M}_{9} d \log \left(z^{2}-w\right)+\mathbb{M}_{10} d \log \left(1-w+w^{2}-z^{2}\right) \\
& +\mathbb{M}_{11} d \log \left(1-3 w+w^{2}+z^{2}\right)+\mathbb{M}_{12} d \log \left(z^{2}-w^{2}-w z^{2}+w^{2} z^{2}\right)
\end{aligned}
$$

for the master integrals in the non-planar integral family, defined in eqs. (2.3) and (2.5):

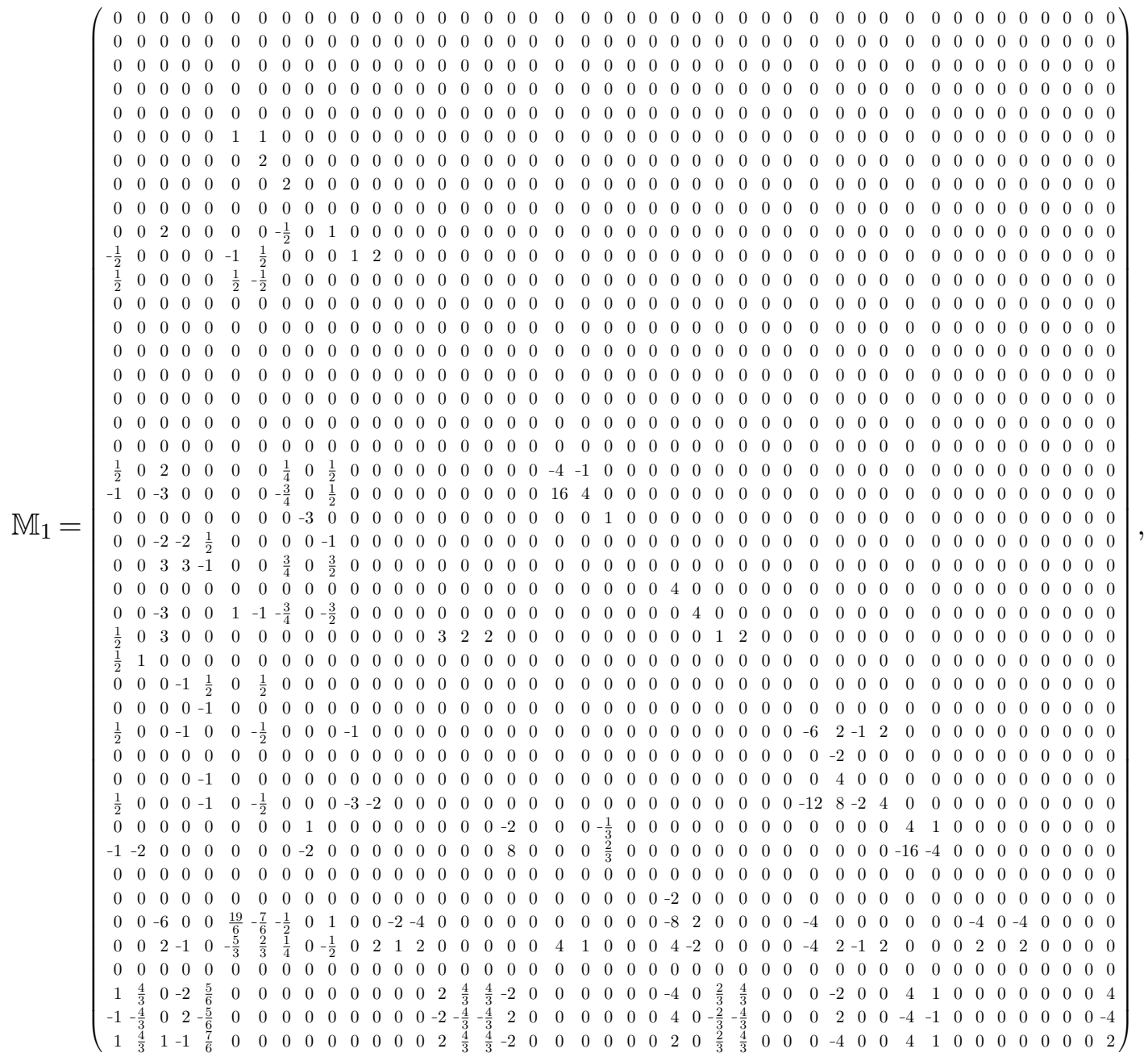




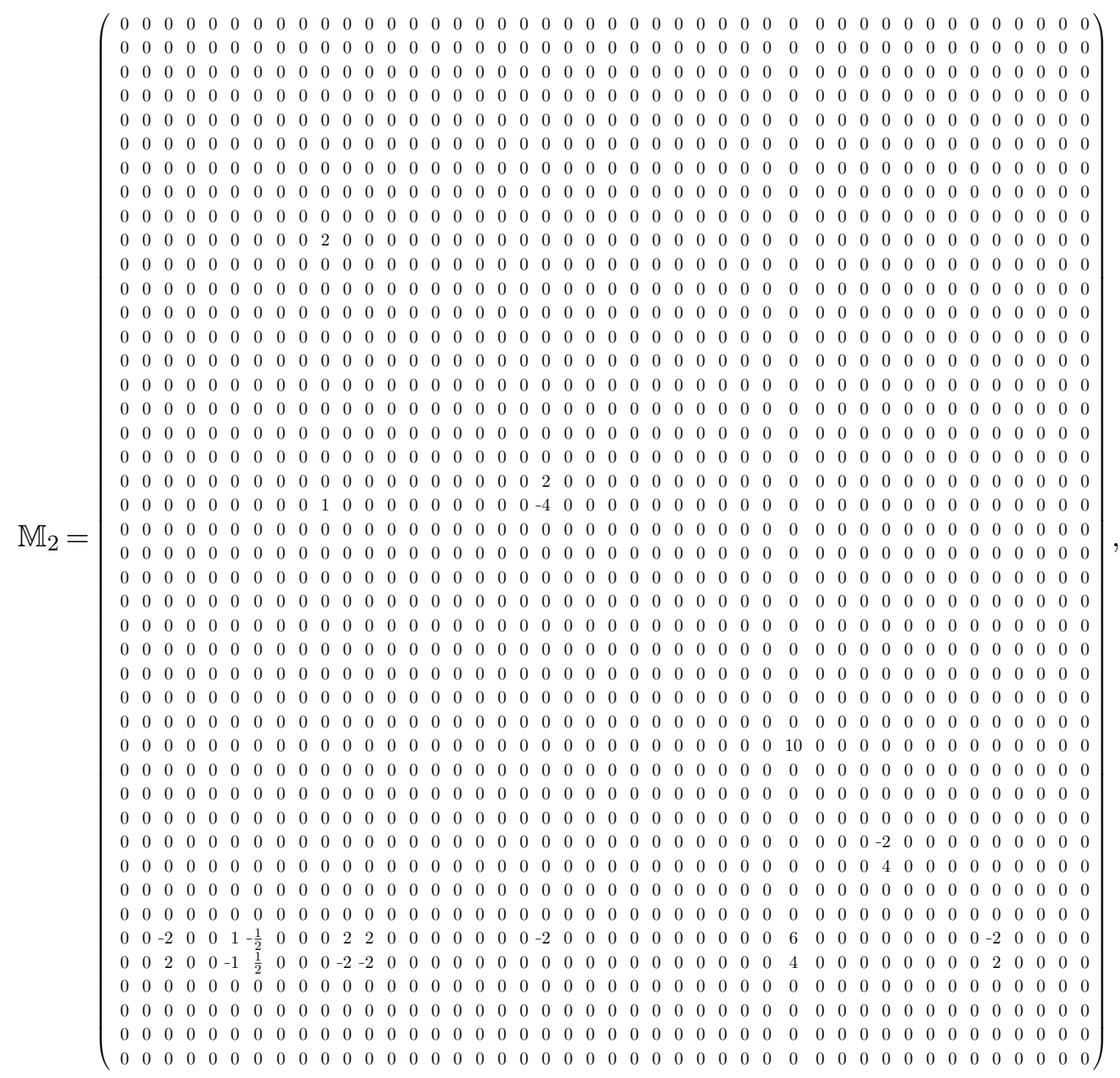




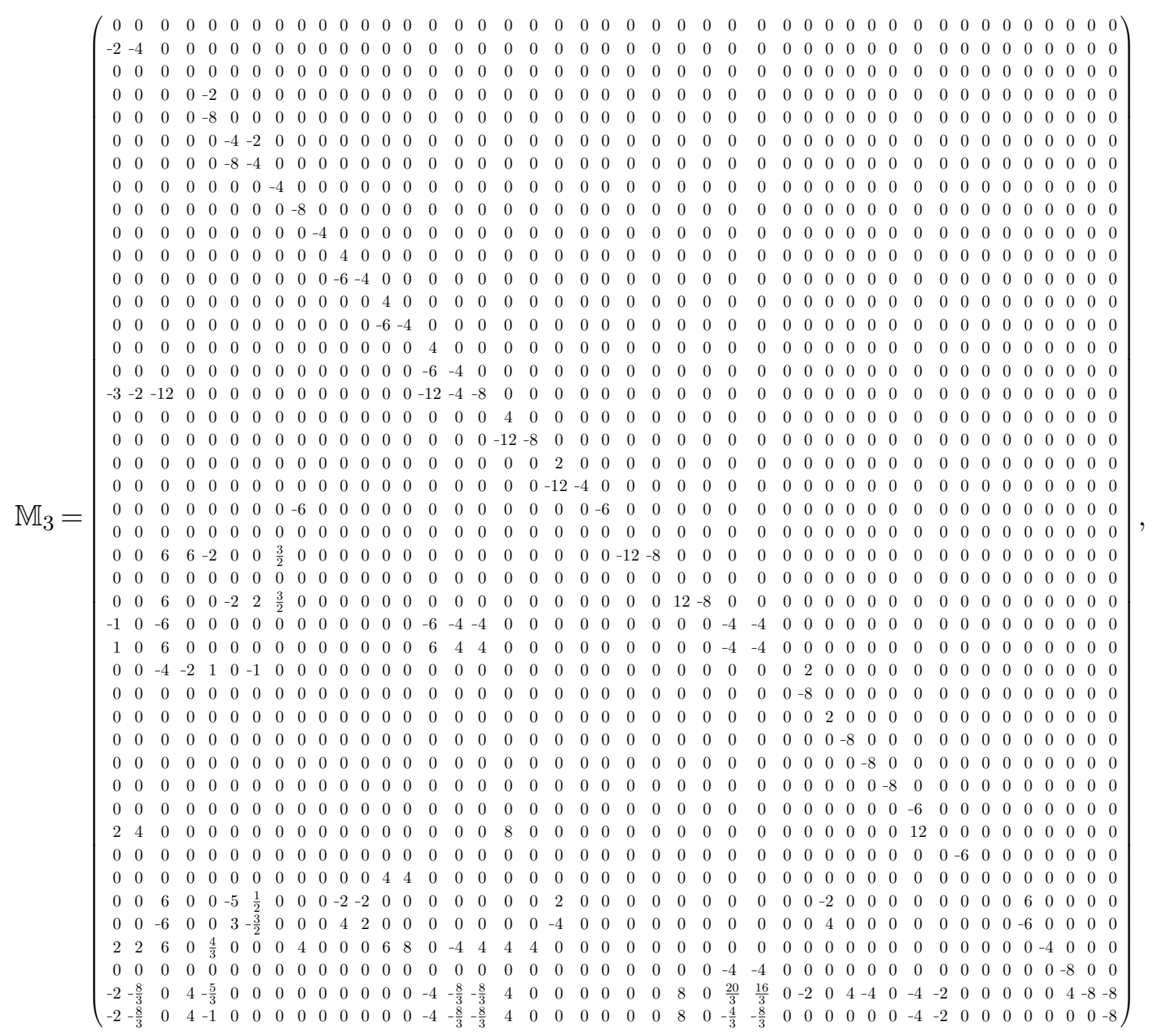

(B.4) 


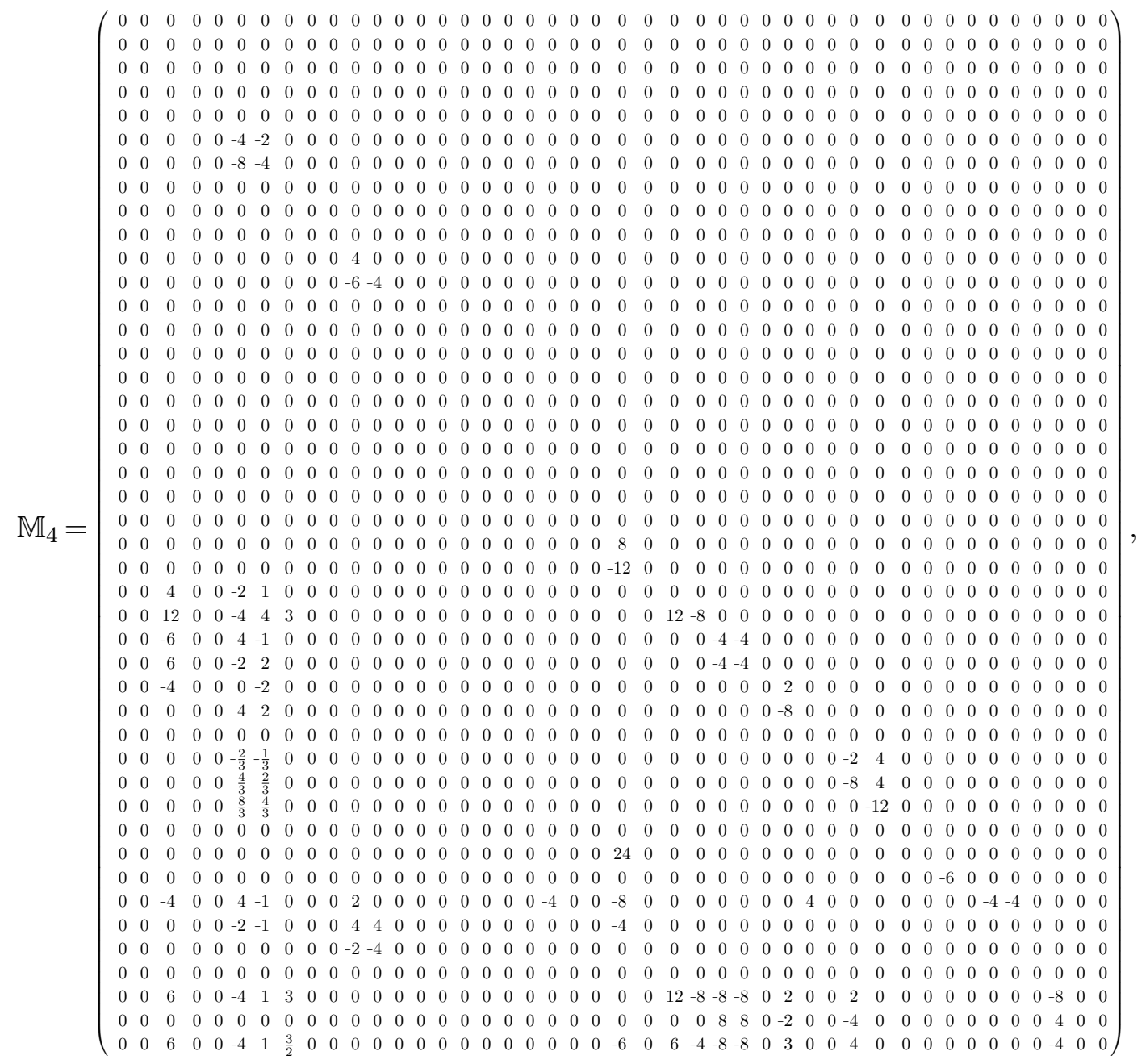




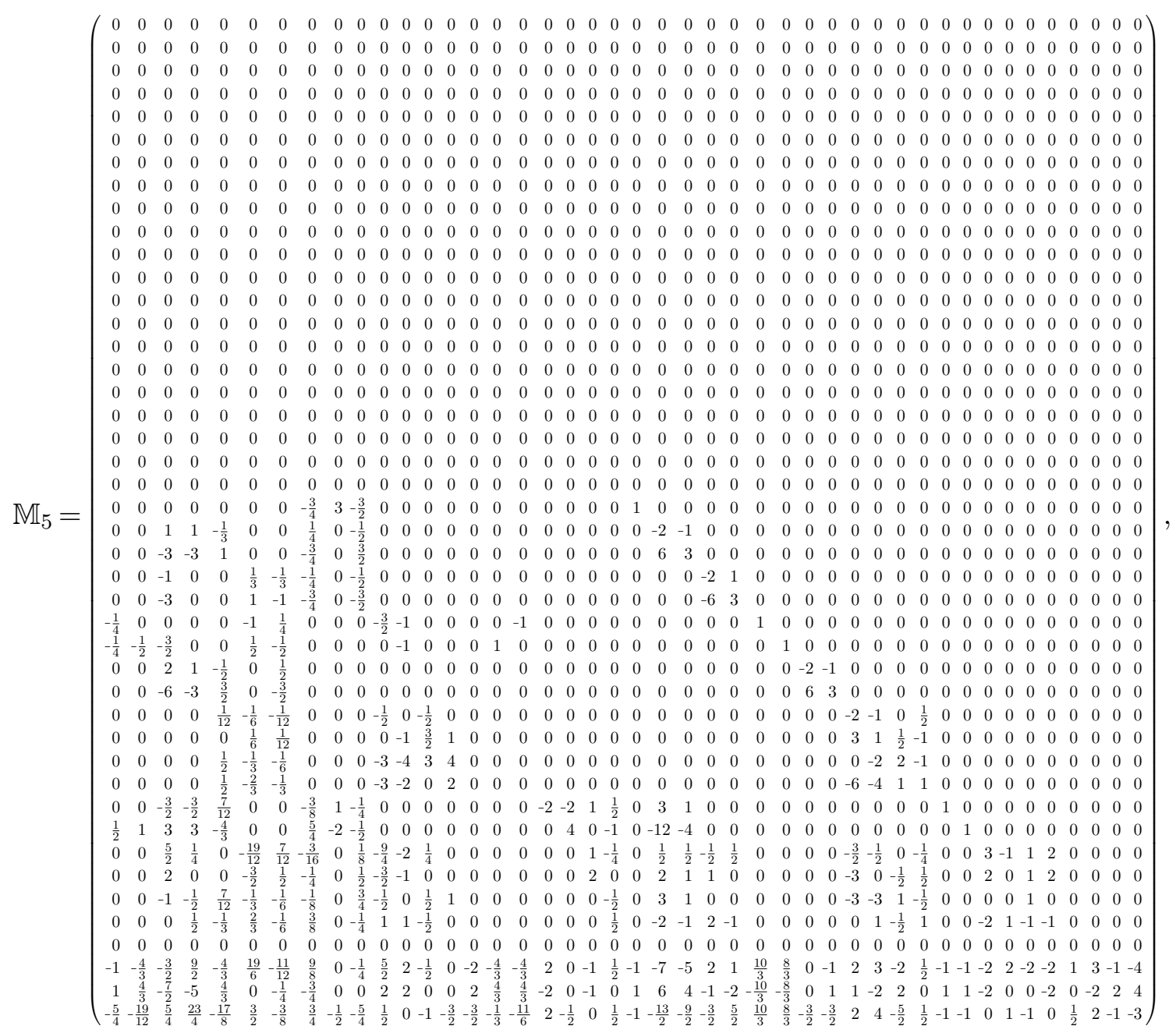




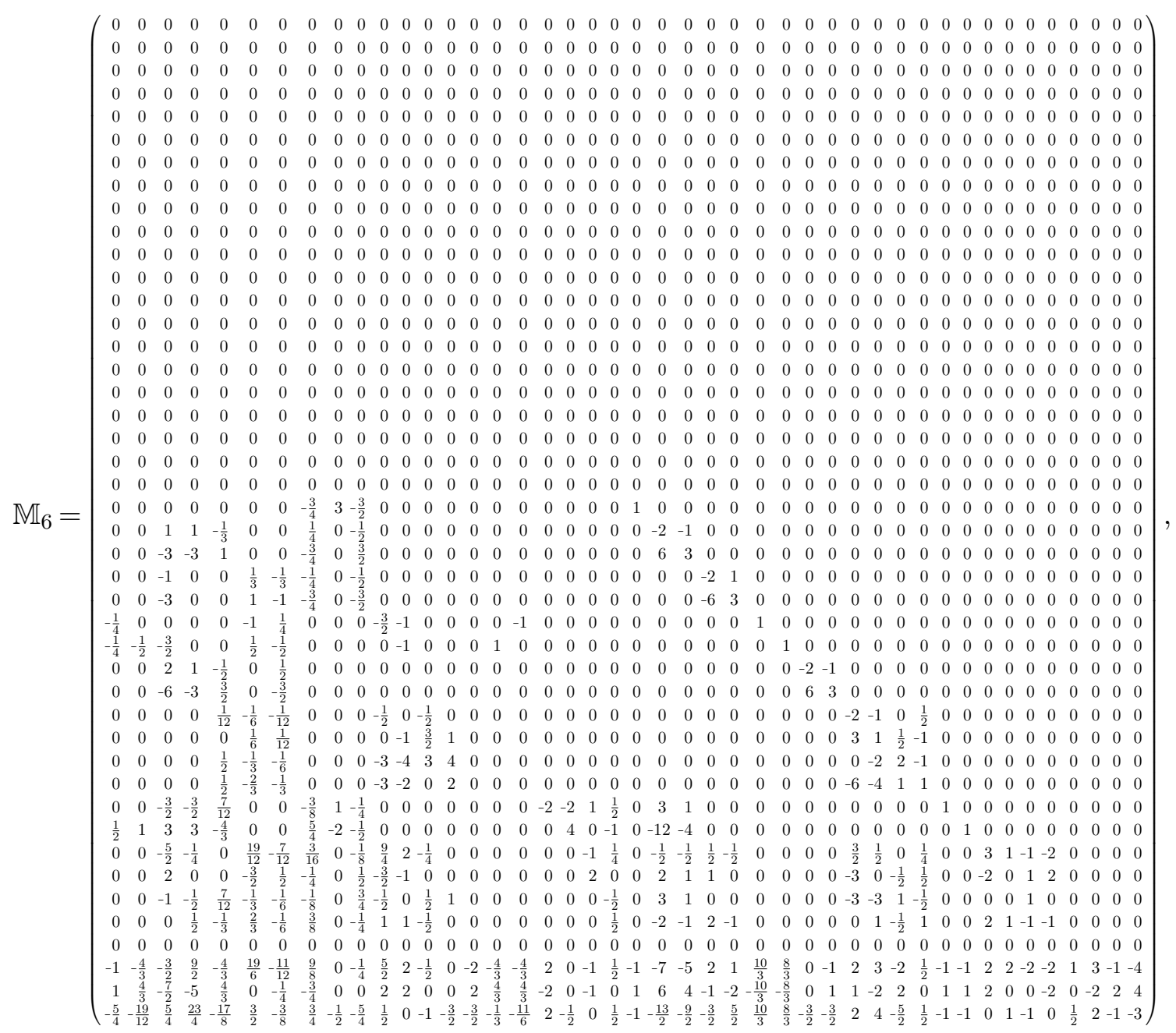




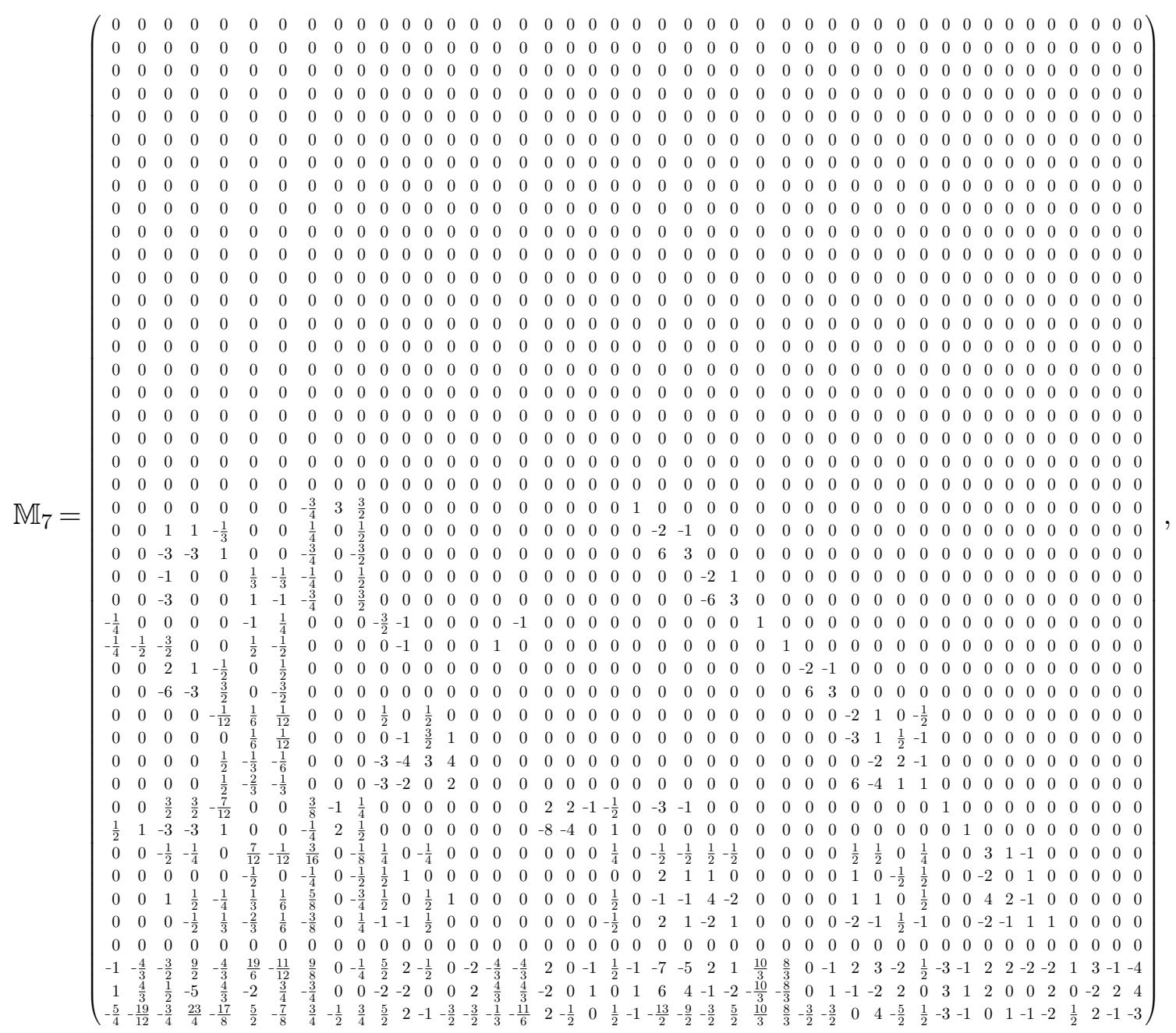




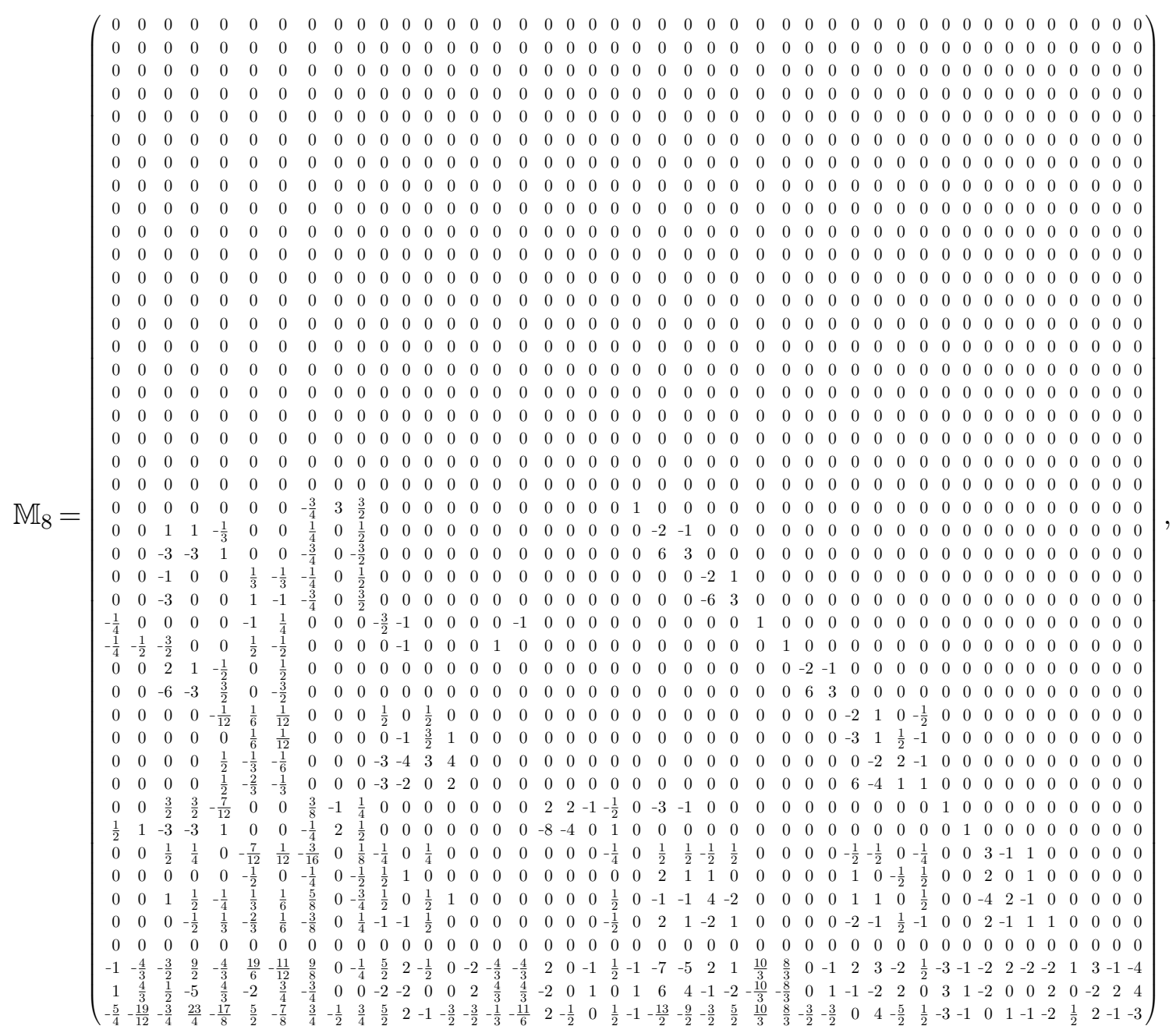




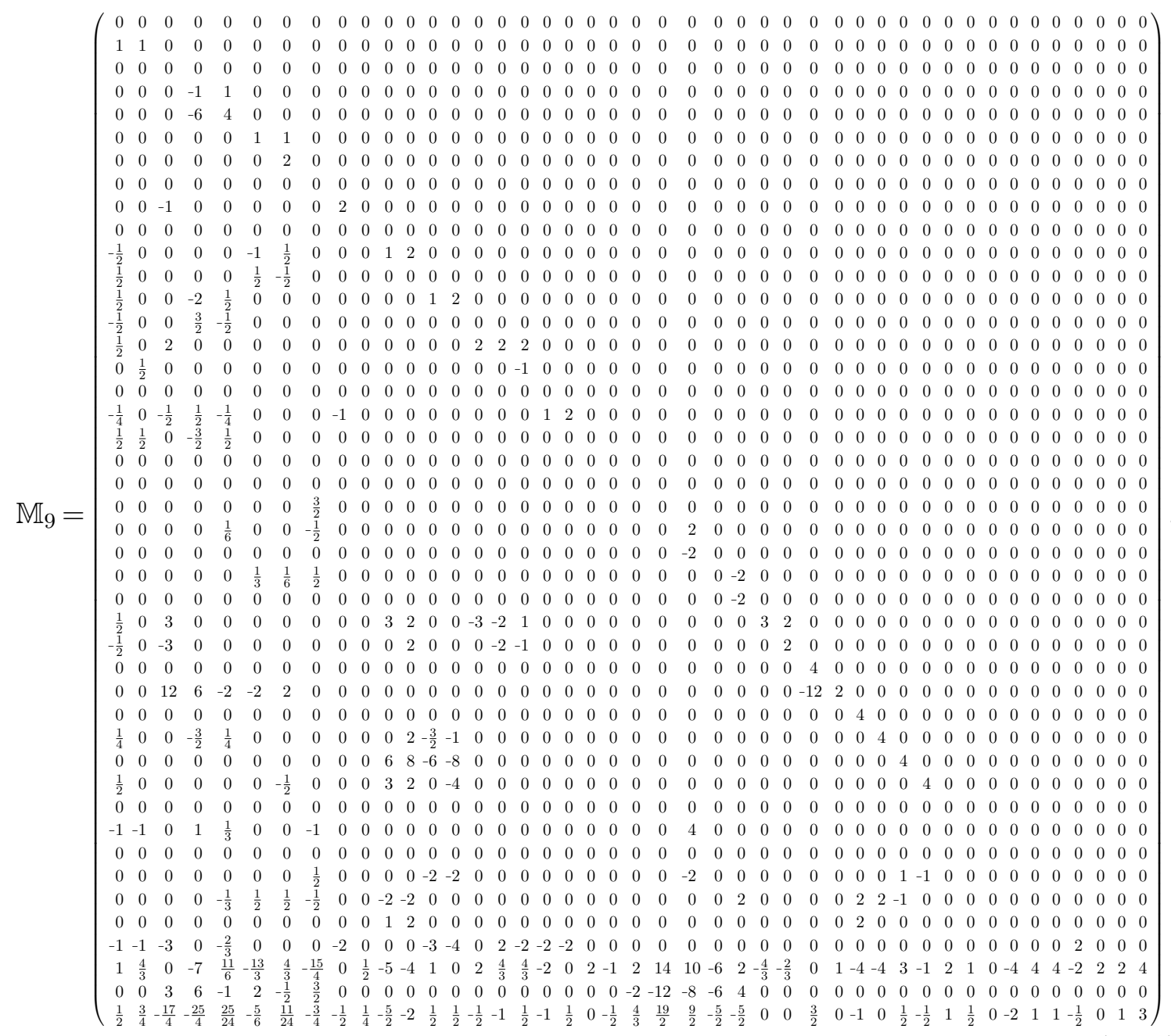




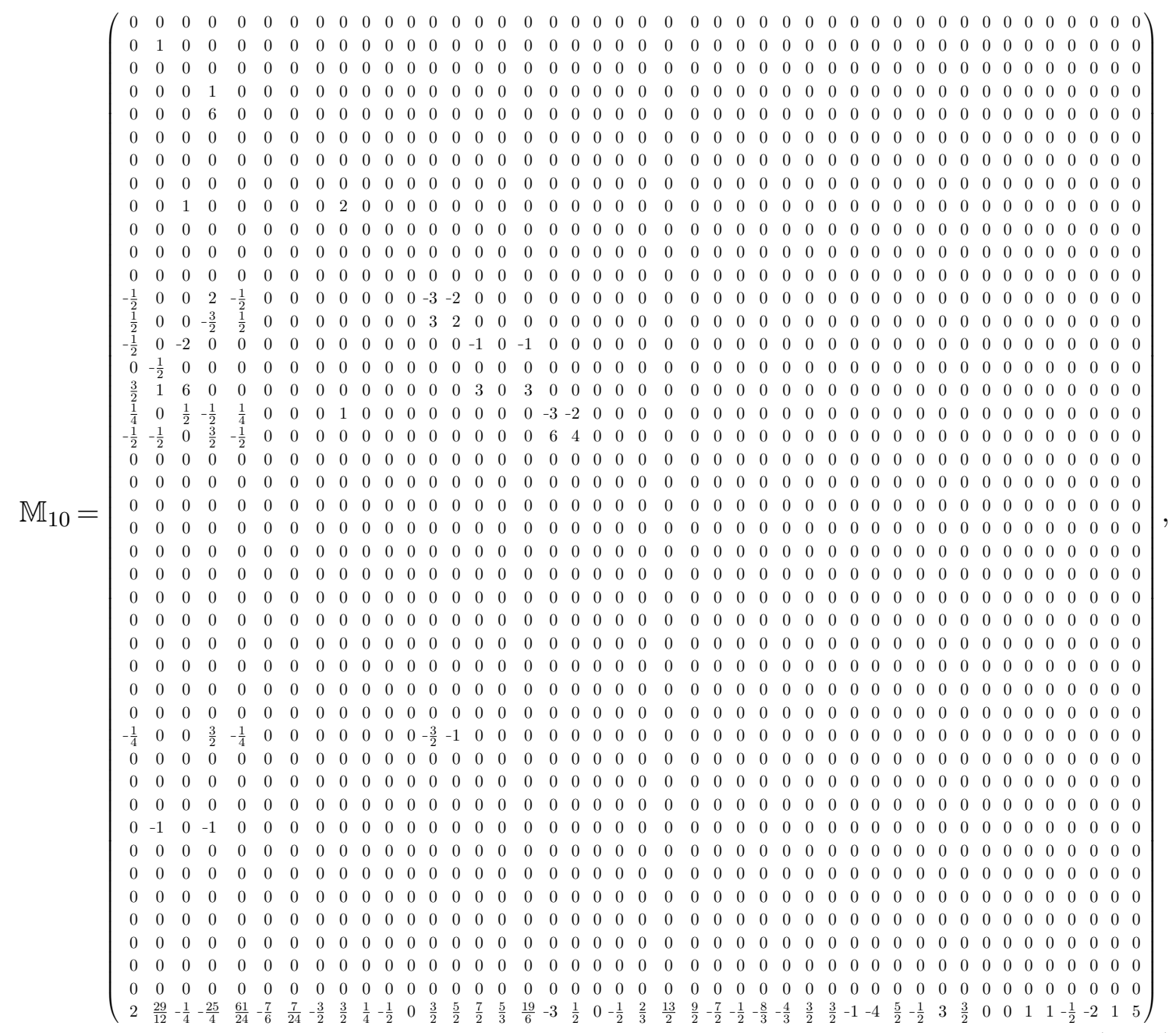

(B.11) 


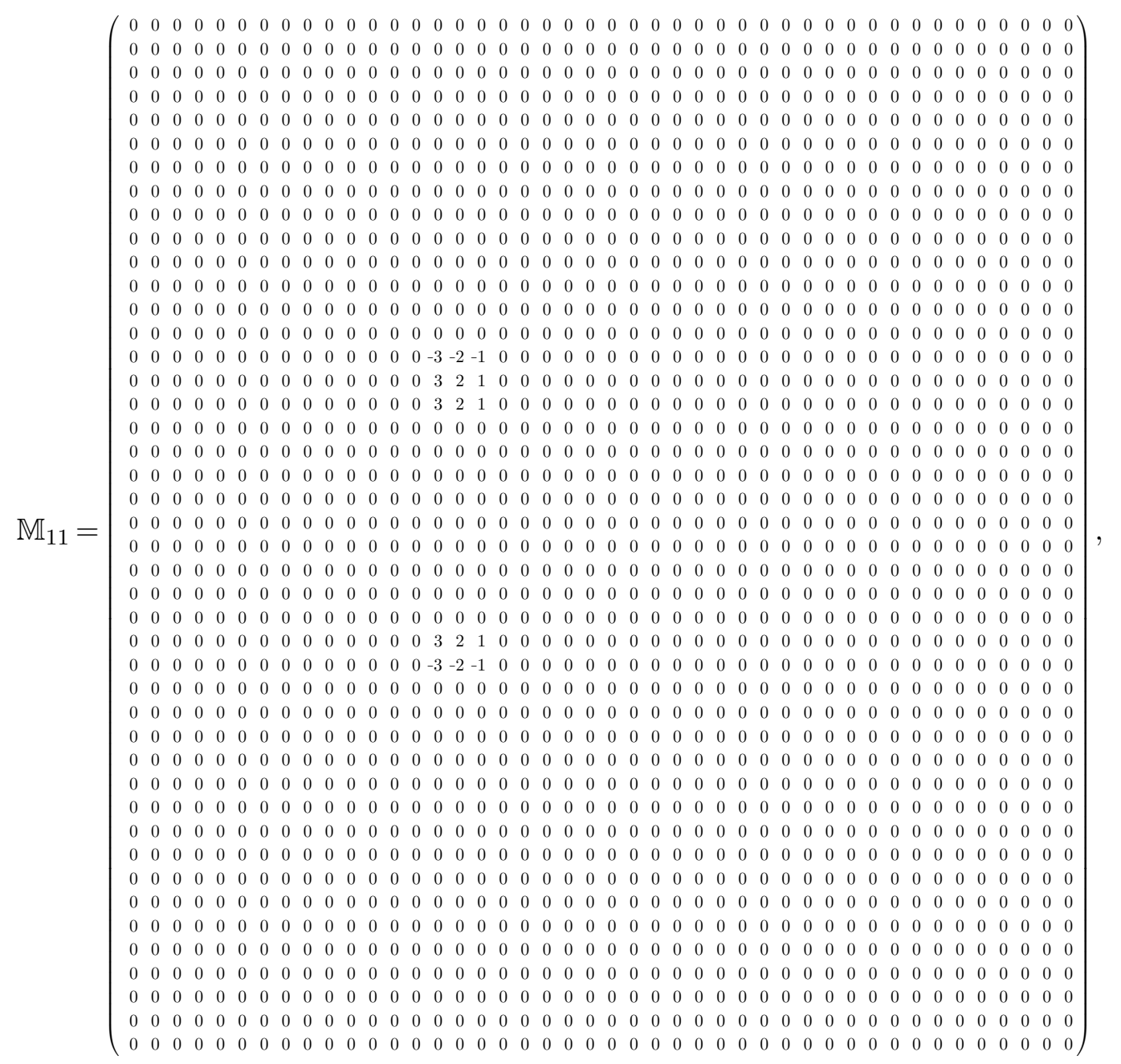

(B.12) 


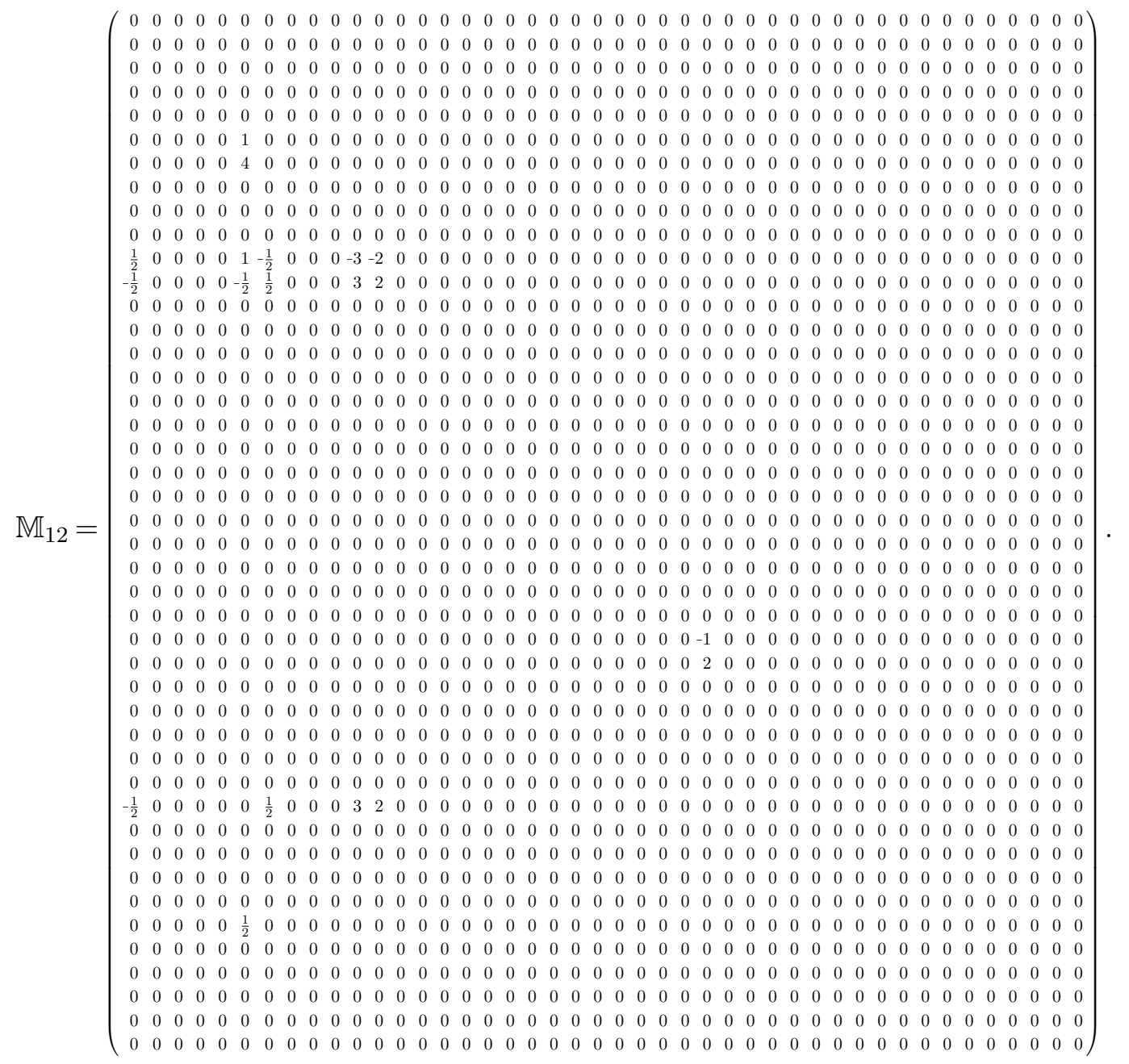

(B.13) 
Open Access. This article is distributed under the terms of the Creative Commons Attribution License (CC-BY 4.0), which permits any use, distribution and reproduction in any medium, provided the original author(s) and source are credited.

\section{References}

[1] P. Mastrolia, M. Passera, A. Primo and U. Schubert, Master integrals for the NNLO virtual corrections to $\mu$ e scattering in QED: the planar graphs, JHEP 11 (2017) 198 [arXiv: 1709.07435] [INSPIRE].

[2] P. Mastrolia, M. Passera, A. Primo, U. Schubert and W.J. Torres Bobadilla, On

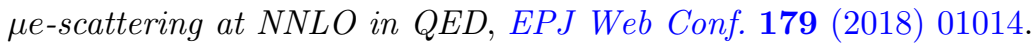

[3] C.M. Carloni Calame, M. Passera, L. Trentadue and G. Venanzoni, A new approach to evaluate the leading hadronic corrections to the muon g-2, Phys. Lett. B 746 (2015) 325 [arXiv: 1504.02228] [INSPIRE].

[4] G. Abbiendi et al., Measuring the leading hadronic contribution to the muon g-2 via $\mu$ e scattering, Eur. Phys. J. C 77 (2017) 139 [arXiv:1609.08987] [INSPIRE].

[5] F. Jegerlehner, The Anomalous Magnetic Moment of the Muon, Springer Tracts Mod. Phys. 274 (2017) 1 .

[6] Belle, Belle II collaborations, T. Ferber and B. Schwartz, Perspectives of a precise measurement of the charge asymmetry in muon pair production at Belle II, J. Univ. Sci. Tech. China 46 (2016) 476 [INSPIRE].

[7] A. Aleksejevs, S. Barkanova and V. Zykunov, NLO electroweak radiative corrections for four-fermionic process at Belle II, EPJ Web Conf. 138 (2017) 06001 [arXiv:1701.07047] [INSPIRE].

[8] F. Ignatov, Status of $R(s)$ measurements by energy scan method, EPJ Web Conf. 179 (2018) 01005 .

[9] Z. Bern, L.J. Dixon and A. Ghinculov, Two loop correction to Bhabha scattering, Phys. Rev. D 63 (2001) 053007 [hep-ph/0010075] [INSPIRE].

[10] Working Group on Radiative Corrections and Monte Carlo Generators for Low ENERGIES collaborations, S. Actis et al., Quest for precision in hadronic cross sections at low energy: Monte Carlo tools vs. experimental data, Eur. Phys. J. C 66 (2010) 585 [arXiv: 0912.0749] [INSPIRE].

[11] F.V. Tkachov, A Theorem on Analytical Calculability of Four Loop Renormalization Group Functions, Phys. Lett. B 100 (1981) 65 [INSPIRE].

[12] K.G. Chetyrkin and F.V. Tkachov, Integration by Parts: The Algorithm to Calculate $\beta$-functions in 4 Loops, Nucl. Phys. B 192 (1981) 159 [inSPIRE].

[13] S. Laporta, High precision calculation of multiloop Feynman integrals by difference equations, Int. J. Mod. Phys. A 15 (2000) 5087 [hep-ph/0102033] [InSPIRE].

[14] A.V. Kotikov, Differential equations method: New technique for massive Feynman diagrams calculation, Phys. Lett. B 254 (1991) 158 [INSPIRE].

[15] E. Remiddi, Differential equations for Feynman graph amplitudes, Nuovo Cim. A 110 (1997) 1435 [hep-th/9711188] [INSPIRE]. 
[16] T. Gehrmann and E. Remiddi, Differential equations for two loop four point functions, Nucl. Phys. B 580 (2000) 485 [hep-ph/9912329] [INSPIRE].

[17] J.M. Henn, Multiloop integrals in dimensional regularization made simple, Phys. Rev. Lett. 110 (2013) 251601 [arXiv:1304.1806] [INSPIRE].

[18] M. Argeri et al., Magnus and Dyson Series for Master Integrals, JHEP 03 (2014) 082 [arXiv: 1401.2979] [INSPIRE].

[19] S. Di Vita, P. Mastrolia, U. Schubert and V. Yundin, Three-loop master integrals for ladder-box diagrams with one massive leg, JHEP 09 (2014) 148 [arXiv:1408.3107] [INSPIRE].

[20] R. Bonciani, S. Di Vita, P. Mastrolia and U. Schubert, Two-Loop Master Integrals for the mixed EW-QCD virtual corrections to Drell-Yan scattering, JHEP 09 (2016) 091 [arXiv: 1604.08581] [INSPIRE].

[21] S. Di Vita, P. Mastrolia, A. Primo and U. Schubert, Two-loop master integrals for the leading QCD corrections to the Higgs coupling to a $W$ pair and to the triple gauge couplings $Z W W$ and $\gamma^{*} W W, J H E P 04$ (2017) 008 [arXiv: 1702.07331] [INSPIRE].

[22] A. Goncharov, Polylogarithms in arithmetic and geometry, Proc. ICM 1-2 (1995) 374.

[23] E. Remiddi and J.A.M. Vermaseren, Harmonic polylogarithms, Int. J. Mod. Phys. A 15 (2000) 725 [hep-ph/9905237] [INSPIRE].

[24] T. Gehrmann and E. Remiddi, Numerical evaluation of harmonic polylogarithms, Comput. Phys. Commun. 141 (2001) 296 [hep-ph/0107173] [INSPIRE].

[25] J. Vollinga and S. Weinzierl, Numerical evaluation of multiple polylogarithms, Comput. Phys. Commun. 167 (2005) 177 [hep-ph/0410259] [INSPIRE].

[26] A. von Manteuffel and C. Studerus, Reduze 2 - Distributed Feynman Integral Reduction, arXiv: 1201.4330 [INSPIRE].

[27] C.W. Bauer, A. Frink and R. Kreckel, Introduction to the GiNaC framework for symbolic computation within the C++ programming language, J. Symb. Comput. 33 (2000) 1 [cs/0004015] [INSPIRE].

[28] S. Borowka, G. Heinrich, S.P. Jones, M. Kerner, J. Schlenk and T. Zirke, SecDec-3.0: numerical evaluation of multi-scale integrals beyond one loop, Comput. Phys. Commun. 196 (2015) 470 [arXiv : 1502.06595] [INSPIRE].

[29] A. von Manteuffel, E. Panzer and R.M. Schabinger, A quasi-finite basis for multi-loop Feynman integrals, JHEP 02 (2015) 120 [arXiv:1411.7392] [INSPIRE].

[30] R. Bonciani, P. Mastrolia and E. Remiddi, Vertex diagrams for the QED form-factors at the two loop level, Nucl. Phys. B 661 (2003) 289 [Erratum ibid. B 702 (2004) 359] [hep-ph/0301170] [INSPIRE].

[31] P. Mastrolia and E. Remiddi, Two loop form-factors in QED, Nucl. Phys. B 664 (2003) 341 [hep-ph/0302162] [INSPIRE].

[32] R. Bonciani, P. Mastrolia and E. Remiddi, QED vertex form-factors at two loops, Nucl. Phys. B 676 (2004) 399 [hep-ph/0307295] [INSPIRE].

[33] M. Czakon, Tops from Light Quarks: Full Mass Dependence at Two-Loops in QCD, Phys. Lett. B 664 (2008) 307 [arXiv:0803.1400] [InSPIRE]. 
[34] M. Czakon and A. Mitov, NNLO corrections to top pair production at hadron colliders: the quark-gluon reaction, JHEP 01 (2013) 080 [arXiv: 1210.6832] [INSPIRE].

[35] M. Czakon and A. Mitov, NNLO corrections to top-pair production at hadron colliders: the all-fermionic scattering channels, JHEP 12 (2012) 054 [arXiv:1207.0236] [INSPIRE].

[36] P. Bärnreuther, M. Czakon and A. Mitov, Percent Level Precision Physics at the Tevatron: First Genuine NNLO QCD Corrections to $q \bar{q} \rightarrow t \bar{t}+X$, Phys. Rev. Lett. 109 (2012) 132001 [arXiv: 1204.5201] [INSPIRE].

[37] M. Czakon, P. Fiedler and A. Mitov, Total Top-Quark Pair-Production Cross Section at Hadron Colliders Through $O\left(\alpha_{S}^{4}\right)$, Phys. Rev. Lett. 110 (2013) 252004 [arXiv:1303.6254] [INSPIRE].

[38] R. Bonciani, A. Ferroglia, T. Gehrmann, D. Maître and C. Studerus, Two-Loop Fermionic Corrections to Heavy-Quark Pair Production: The Quark-Antiquark Channel, JHEP 07 (2008) 129 [arXiv:0806.2301] [InSPIRE].

[39] R. Bonciani, A. Ferroglia, T. Gehrmann and C. Studerus, Two-Loop Planar Corrections to Heavy-Quark Pair Production in the Quark-Antiquark Channel, JHEP 08 (2009) 067 [arXiv:0906.3671] [INSPIRE].

[40] R. Bonciani, A. Ferroglia, T. Gehrmann, A. von Manteuffel and C. Studerus, Two-Loop Leading Color Corrections to Heavy-Quark Pair Production in the Gluon Fusion Channel, JHEP 01 (2011) 102 [arXiv: 1011.6661] [INSPIRE].

[41] A. von Manteuffel and C. Studerus, Massive planar and non-planar double box integrals for light $N_{f}$ contributions to $g g \rightarrow t \bar{t}$, JHEP 10 (2013) 037 [arXiv:1306.3504] [INSPIRE].

[42] R. Bonciani, A. Ferroglia, T. Gehrmann, A. von Manteuffel and C. Studerus, Light-quark two-loop corrections to heavy-quark pair production in the gluon fusion channel, JHEP 12 (2013) 038 [arXiv: 1309.4450] [INSPIRE].

[43] L. Adams, E. Chaubey and S. Weinzierl, Analytic results for the planar double box integral relevant to top-pair production with a closed top loop, arXiv:1806.04981 [INSPIRE].

[44] A. von Manteuffel and L. Tancredi, A non-planar two-loop three-point function beyond multiple polylogarithms, JHEP 06 (2017) 127 [arXiv:1701.05905] [INSPIRE].

[45] X. Liu, Y.-Q. Ma and C.-Y. Wang, A Systematic and Efficient Method to Compute Multi-loop Master Integrals, Phys. Lett. B 779 (2018) 353 [arXiv:1711.09572] [INSPIRE].

[46] O.V. Tarasov, Connection between Feynman integrals having different values of the space-time dimension, Phys. Rev. D 54 (1996) 6479 [hep-th/9606018] [INSPIRE].

[47] R.N. Lee, Space-time dimensionality D as complex variable: Calculating loop integrals using dimensional recurrence relation and analytical properties with respect to D, Nucl. Phys. B 830 (2010) 474 [arXiv:0911.0252] [INSPIRE].

[48] R.N. Lee, Presenting LiteRed: a tool for the Loop InTEgrals REDuction, arXiv:1212.2685 [INSPIRE].

[49] R.N. Lee, LiteRed 1.4: a powerful tool for reduction of multiloop integrals, J. Phys. Conf. Ser. 523 (2014) 012059 [arXiv: 1310.1145] [INSPIRE]. 